\title{
Diffuse interface modeling of three-phase contact line dynamics on curved boundaries: A lattice Boltzmann model for large density and viscosity ratios
}

\author{
Abbas Fakhari ${ }^{\mathrm{a}}$, Diogo Bolster ${ }^{\mathrm{a}}$ \\ ${ }^{a}$ Department of Civil and Environmental Engineering and Earth Sciences \\ University of Notre Dame, Notre Dame, IN 46556, USA
}

\begin{abstract}
We introduce a simple and efficient lattice Boltzmann method for immiscible multiphase flows, capable of handling large density and viscosity contrasts. The model is based on a diffuse-interface phase-field approach. Within this context we propose a new algorithm for specifying the threephase contact angle on curved boundaries within the framework of structured Cartesian grids. The proposed method has superior computational accuracy compared with the common approach of approximating curved boundaries with stair cases. We test the model by applying it to four benchmark problems: (i) wetting and dewetting of a droplet on a flat surface and (ii) on a cylindrical surface, (iii) multiphase flow past a circular cylinder at an intermediate Reynolds number, and (iv) a droplet falling on hydrophilic and superhydrophobic circular cylinders under differing conditions. Where available, our results show good agreement with analytical solutions and/or existing experimental data, highlighting strengths of this new approach.
\end{abstract}

Email address: afakhari@nd.edu (Abbas Fakhari)

Preprint submitted to Journal of Computational Physics

November 7, 2016

(C) 2017. This manuscript version is made available under the Elsevier user license http://www.elsevier.com/open-access/userlicense/1.0/ 
Keywords: contact angle, contact line dynamics, curved boundary, lattice Boltzmann method, superhydrophobic surface

2

\section{Introduction}

Fluid-solid interactions are ubiquitous in nature. Common examples of practical interest include flow through porous media, aerodynamics, flow around structures, and colloidal suspensions, to name a few [1]. Within this context, three-phase (solid-liquid-gas) contact line dynamics is a challenging topic of significant global research interest with scientific and engineering applications spanning self-cleaning materials, oil and gas recovery, geological carbon sequestration, groundwater contamination by non aqueous phase liquids, inkjet printers, microfluidics, etc. [2]. While of critical importance, simulating such systems still poses challenges in terms of accuracy, efficiency, and numerical stability.

Various numerical approaches to tackle this problem exist [3]. Within these, diffuse interface models [4-9] present a promising framework for studying three-phase moving contact line dynamics. In part, this is due to their intrinsic capacity to handle the singularity at the three-phase contact point while not requiring a velocity-slip model on the solid surface. Among diffusive interface models, phase-field models are a popular choice, wherein the interface between different phases is usually obtained by solving a Cahn-Hilliard like equation [10] or a conservative phase-field equation [11, 12].

The interface tracking equation in phase-field models can be solved by any number of numerical methods, including finite differences or finite volumes [13], as well as Lattice Boltzmann Methods (LBM) [14-18], the focus of 
this work here. Ease of implementation, straightforward handling of complex boundaries, highly scalable parallelization, and not having to solve an elliptical Poisson (pressure) equation are among the main reasons behind LBM's success [19]. The fact that interphase boundaries are essentially mesoscopic in nature [8] makes LBM, a mesoscopic approach based on kinetic theory of dense fluids [14-16], a powerful candidate for dealing with interfacial phenomena in general and for three-phase contact line dynamics in particular.

Over the past three decades, several LB models for multiphase flows have been proposed in the literature, including the color model [20, 21], the pseudo-potential model [22-25], the free-energy model [26-28], and the mean-field model $[29,30]$. All these models, in their primitive forms, share an undesired feature: the inability to model multiphase systems with large density ratios. From first principles, He and Doolen [16] rigorously connected kinetic theory and LBM for multiphase flows. They then evaluated and identified weaknesses of the aforementioned models. This improved thermodynamic understanding enabled more advanced models to emerge [31-34].

Inamuro et al. [31] proposed an LB model for multiphase flows with large density ratios; the price for this achievement was the need to solve a time-consuming pressure-Poisson equation and inability to guarantee mass conservation due to using a cut-off value in updating of density. Lee and Lin [32] proposed a three-step (pre-streaming collision, streaming, and poststreaming collision) LBM by invoking the potential form of the surfacetension force in the interface tracking formula and the stress form in the momentum equation, as had been proposed by Jamet et al. [7]. Using a combination of central and biased finite-difference schemes, which is shown 
to compromise mass conservation [35], they achieved numerical stability at high density ratios. The main criticism of this model is its complicated and inefficient algorithm. Later it was shown that the three-step algorithm could be reduced to the conventional, two-step collision-streaming without losing accuracy or stability [36]. Zheng et al. [37] proposed a modified freeenergy-based LBM for multiphase flows intended for high density ratios, but it has been shown that it is limited to density-matched fluids [38]. Fakhari and Rahimian [38] presented a phase-field LBM for binary fluids at high Reynolds numbers which is limited to moderate density ratios. Based on the Cahn-Hilliard diffuse interface model [13], Lee and Liu [36] presented an improved version of the free-energy model, which was later extended to multiphase flows at high Reynolds numbers [39]. However, the inconsistent use of central and biased finite differences means that these models [36, 39] can violate mass and momentum conservation [40]. Mass conservation was remedied in Ref. [41] but momentum conservation issues remain.

Of important note to our problem of interest is that most of the aforementioned studies only consider multiphase flow in the absence of solid boundaries or with neutral wall boundary conditions at best [31-33, 38]. Unlike immersed boundary methods, which are readily used to tackle curved boundaries on Cartesian grids [42], simulation of three-phase contact line motion on curved solid boundaries is still a challenging subject in LBM. The color model is widely used to this end [34], most likely due to its efficient implementation. Despite this, even with improvements over time, the color model has had limited capabilities in dealing with realistic multiphase flows with large density differences [43], with a recent exception [44]. Additionally, the 
resulting flow field can have very large so-called spurious currents or parasitic velocities, which are unphysical and potentially undesirable artifacts. In order to control the spurious velocities in the color model, Leclaire et al. [45] proposed a relationship for scaling the interfacial thickness with grid resolution. Also, some correction terms can be added to improve the numerical stability and accuracy of the color model for simulation of two-layer Poiseuille flow at high density and viscosity ratios $[44,46]$. The pseudopotential model shares similar drawbacks with even larger spurious velocities reported [47]. The mean-field model [30] is in our view more appealing as it has a strong physical foundation based on kinetic theory for dense fluids and is versatile in handling complex flow phenomena with rapid topological changes such as interface disintegration and coalescence [48]. Improved versions of the mean-field model are even more practical particularly for simulation of multiphase flows at high density ratios [36, 41], at high Reynolds numbers [38, 39], for thermocapillary fluids [49], and most importantly for this work with fluid-solid interactions [50].

Although wettability effects on a flat surface have been considered in some previous LB studies [36], for those cases the solid boundaries had to be co-located with lattice nodes, restricting their application to perfectly horizontal or vertical flat walls. In some other LB models, curved surfaces are approximated by stair-cased line segments in 2D (plain segments in 3D), potentially reducing the physical accuracy of the simulations. For example, in a recent color model [34], the actual wall location is only considered for constructing the color variable at the solid boundaries, and no treatment is performed for the distribution functions. Also, the model in Ref. [50] re- 
quires significant care in dealing with boundary nodes and spatial derivatives at solid boundaries. It can also suffer violation of conservation laws.

In this work, we introduce a simple, yet robust, LB model for multiphase flows at high density ratios. The proposed model does not need biased or mixed finite differences, as are required in previous models for stability reasons [32, 36, 39], and therefore conserves both mass and momentum. Aside from two nonlocal distribution functions, the proposed model requires only one nonlocal variable (the phase field), compared with three to four nonlocal variables in some previous works $[36,39,50]$. This greatly enhances the simplicity and efficiency of the algorithm. We also propose a new algorithm for prescribing the three-phase contact angle on curved boundaries without using a stair-case approximation for the solid boundary. We start by presenting the macroscopic governing equations in Sec. 2, followed by introducing the conservative phase-field LB model in Sec. 3. The new algorithm for handling wetting boundary conditions on curved surfaces is described in Sec. 4. We test the proposed model by running various benchmark problems in Sec. 5 and conclude the paper with a summary in Sec. 6 .

\section{Governing equations}

\subsection{Interface tracking equation}

While most phase-field models rely on Cahn-Hilliard theory [10, 9] for interface tracking, a fourth-order derivative that arises in taking the Laplacian of the chemical potential can cause difficulties and reduce the accuracy and efficiency of numerical approximations. In this study, we use a different form of phase-field equation that contains at most second-order derivatives, which 
is called the conservative phase-field equation [11, 12].

For an incompressible binary-fluid system, we define a phase-field variable $\phi$ in such a way that it is zero in the light fluid and one in the heavy fluid. The following interface tracking equation is used to track the interface between different fluids [12]:

$$
\frac{\partial \phi}{\partial t}+\nabla \cdot(\phi \boldsymbol{u})=\boldsymbol{\nabla} \cdot\left[M\left(\boldsymbol{\nabla} \phi-\frac{4}{\xi} \phi(1-\phi) \hat{\boldsymbol{n}}\right)\right]
$$

where $t$ is time, $\mathbf{u}$ is the macroscopic velocity vector, $M$ is the mobility, $\xi$ is the interfacial thickness, and $\hat{\boldsymbol{n}}$ is the unit vector normal to the interface, with its direction pointing away from the heavy fluid, such that

$$
\hat{\boldsymbol{n}}=\frac{\nabla \phi}{|\nabla \phi|}
$$

In diffuse-interface models, the equilibrium phase-field profile for an interface located at $\boldsymbol{x}_{0}$ is usually assumed to vary according to

$$
\phi(\boldsymbol{x})=\frac{1}{2}\left[1-\tanh \left(\frac{\left|\boldsymbol{x}-\boldsymbol{x}_{0}\right|}{\xi / 2}\right)\right]
$$

which is typically used to set the initial condition on $\phi$.

In the absence of fluid interaction with solid walls, or in dealing with neutral wetting conditions (i.e. a $90^{\circ}$ contact angle), Neumann boundary conditions are applied $\left.\hat{\boldsymbol{n}}_{\mathrm{w}} \cdot \boldsymbol{\nabla} \phi\right|_{\boldsymbol{x}_{\mathrm{w}}}=0$, where $\hat{\boldsymbol{n}}_{\mathrm{w}}$ is the unit vector normal to, and outwards from, the solid wall. To impose a specified contact angle at a solid boundary, the following boundary condition has been proposed [6]:

$$
\left.\hat{\boldsymbol{n}}_{\mathrm{w}} \cdot \nabla \phi\right|_{\boldsymbol{x}_{\mathrm{w}}}=\Theta \phi_{\mathrm{w}}\left(1-\phi_{\mathrm{w}}\right)
$$

where $\phi_{\mathrm{w}}$ is the phase-field value at wall and $\Theta$ is related to the equilibrium contact angle $\theta$ by

$$
\Theta=-\sqrt{\frac{2 \beta}{\kappa}} \cos \theta
$$


where coefficients $\beta$ and $\kappa$ are related to the surface tension $\sigma$ and interfacial thickness $\xi$ by $\beta=12 \sigma / \xi$ and $\kappa=3 \sigma \xi / 2$.

Note that there are underlying differences between the present approach and other models for specifying the contact angle. The majority of previous studies use a geometric formula [51] to impose the wetting boundary conditions. In addition to producing larger parasitic currents, the geometric approach works only on flat walls where the normal to the surface aligns with lattice nodes. On the other hand, specifying a potential, similar to the one in Eq. (4), to model the interaction of different fluids with a solid surface is more consistent with the nature of phase-field models $[5,6]$.

\subsection{Navier-Stokes equations}

The continuity and momentum equations that govern isothermal, incompressible, multiphase flows are

$$
\begin{aligned}
\frac{\partial \rho}{\partial t}+\nabla \cdot \rho \boldsymbol{u} & =0 \\
\rho\left(\frac{\partial \boldsymbol{u}}{\partial t}+\boldsymbol{u} \cdot \boldsymbol{\nabla} \boldsymbol{u}\right) & =-\boldsymbol{\nabla} p+\nabla \cdot\left(\mu\left[\boldsymbol{\nabla} \boldsymbol{u}+(\boldsymbol{\nabla} \boldsymbol{u})^{T}\right]\right)+\boldsymbol{F}_{\mathrm{s}}+\boldsymbol{F}_{\mathrm{b}}
\end{aligned}
$$

where $\rho$ and $\mu$ are the local fluid density and viscosity, respectively, $p$ is the macroscopic pressure, $\boldsymbol{F}_{\mathrm{b}}$ is the body force, and $\boldsymbol{F}_{\mathrm{s}}$ is the surface tension force, which can take the following form [5]:

$$
\boldsymbol{F}_{\mathrm{s}}=\mu_{\phi} \nabla \phi
$$

where

$$
\mu_{\phi}=4 \beta \phi(\phi-1)(\phi-1 / 2)-\kappa \nabla^{2} \phi
$$

is the chemical potential for binary fluids. 


\section{Multiphase flow solver: lattice Boltzmann method}

\subsection{LBE for interface tracking}

The following LBE has previously been shown to recover Eq. (1) for the conservative phase field [52]:

$$
h_{\alpha}\left(\boldsymbol{x}+\boldsymbol{e}_{\alpha} \delta t, t+\delta t\right)=h_{\alpha}(\boldsymbol{x}, t)-\frac{h_{\alpha}(\boldsymbol{x}, t)-h_{\alpha}^{\mathrm{eq}}(\boldsymbol{x}, t)}{\tau_{\phi}+1 / 2}
$$

where $h_{\alpha}$ is the phase-field distribution function, $\tau_{\phi}$ is the phase-field relaxation time, and $\boldsymbol{e}_{\alpha}$ is the microscopic velocity set. For the D2Q9 lattice, which is used in this study [15]

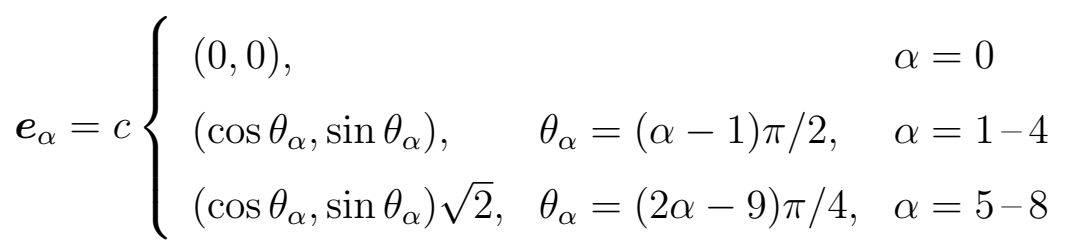

where $c=\delta x / \delta t=1$ and $\delta x$ and $\delta t$ are the lattice length scale and time scale, respectively (on uniform grids $\delta x=\delta t=1$ ). The equilibrium phase-field distribution function is given by

$$
h_{\alpha}^{\mathrm{eq}}=\phi \Gamma_{\alpha}+w_{\alpha} \frac{M}{c_{s}^{2}}\left[\frac{4}{\xi} \phi(1-\phi)\right]\left(\boldsymbol{e}_{\alpha} \cdot \hat{\boldsymbol{n}}\right)
$$

where

$$
\Gamma_{\alpha}=w_{\alpha}\left[1+\frac{\boldsymbol{e}_{\alpha} \cdot \boldsymbol{u}}{c_{s}^{2}}+\frac{\left(\boldsymbol{e}_{\alpha} \cdot \boldsymbol{u}\right)^{2}}{2 c_{s}^{4}}-\frac{\boldsymbol{u} \cdot \boldsymbol{u}}{2 c_{s}^{2}}\right]
$$

$c_{s}=c / \sqrt{3}$ is the speed of sound in the system and $w_{\alpha}$ is the weight coefficient set, where $w_{0}=4 / 9, w_{1-4}=1 / 9$, and $w_{5-8}=1 / 36$. Mobility $M$ is related to the phase-field relaxation time by

$$
M=\tau_{\phi} c_{s}^{2} \delta t
$$


Equation (9) is usually solved with a two-step collision-streaming approach as

$$
\begin{aligned}
h_{\alpha}^{*}(\boldsymbol{x}, t) & =h_{\alpha}-\left.\frac{h_{\alpha}-h_{\alpha}^{\mathrm{eq}}}{\tau_{\phi}+1 / 2}\right|_{(\boldsymbol{x}, t)} \\
h_{\alpha}\left(\boldsymbol{x}+\boldsymbol{e}_{\alpha} \delta t, t+\delta t\right) & =h_{\alpha}^{*}(\boldsymbol{x}, t)
\end{aligned}
$$

where the asterisk denotes the pre-streaming, or post-collision, state. After the streaming step, the phase field is updated by taking the zeroth moment of the phase-field distribution function

$$
\phi=\sum_{\alpha} h_{\alpha}
$$

and the density $\rho$ is found by a simple linear interpolation

$$
\rho=\rho_{\mathrm{L}}+\phi\left(\rho_{\mathrm{H}}-\rho_{\mathrm{L}}\right)
$$

where $\rho_{\mathrm{L}}$ and $\rho_{\mathrm{H}}$ are the densities of the light and heavy fluids, respectively.

It is worth noting that, aside from advantages in computational cost and efficiency, the conservative phase-field equation is much less dispersive when compared with the Cahn-Hilliard equation, particularly when implemented within the LBM framework. This lower-dispersion error hinders the phasefield variable from noticeably undershooting its lowest equilibrium value (here $\left.\phi_{\min }=0\right)$. Therefore the density, which is linearly interpolated from the phase-field via Eq. (1), is less prone to unphysically becoming negative and leading to numerical instabilities at high density ratios. This is a key factor in achieving numerically stable solutions with a high density ratio.

\subsection{LBE for hydrodynamics}

Based on the explicit approach proposed by He et al. [29], we use an improved hydrodynamic evolution equation [38] to update the pressure and 
velocity fields. The lattice Boltzmann equation for nearly incompressible multiphase flows can be written as

$$
\bar{g}_{\alpha}\left(\boldsymbol{x}+\boldsymbol{e}_{\alpha} \delta t, t+\delta t\right)=\bar{g}_{\alpha}(\boldsymbol{x}, t)+\Omega_{\alpha}(\mathbf{x}, t)+F_{\alpha}(\mathbf{x}, t)
$$

where $\bar{g}_{\alpha}$ is the modified hydrodynamic distribution function for the nearly incompressible fluids [30, 39], $\Omega_{\alpha}$ is the collision operator, and the forcing term is [41]

$$
F_{\alpha}=\delta t\left[\left(\Gamma_{\alpha}-w_{\alpha}\right)\left(\rho_{\mathrm{H}}-\rho_{\mathrm{L}}\right) c_{s}^{2}+\Gamma_{\alpha} \mu_{\phi}\right]\left(\boldsymbol{e}_{\alpha}-\boldsymbol{u}\right) \cdot \boldsymbol{\nabla} \phi+\delta t \Gamma_{\alpha}\left(\boldsymbol{e}_{\alpha}-\boldsymbol{u}\right) \cdot \boldsymbol{F}_{\mathrm{b}}
$$
collision operator is

$$
\Omega_{\alpha}=\Omega_{\alpha}^{\mathrm{MRT}}=-\mathbf{M}^{-1} \hat{\mathbf{S}} \mathbf{M}\left(\bar{g}_{\alpha}-\bar{g}_{\alpha}^{\mathrm{eq}}\right)
$$

where the modified equilibrium distribution function is defined by

$$
\bar{g}_{\alpha}^{\mathrm{eq}}=g_{\alpha}^{e q}-\frac{1}{2} F_{\alpha}
$$

and

$$
g_{\alpha}^{e q}=p w_{\alpha}+\rho c_{s}^{2}\left(\Gamma_{\alpha}-w_{\alpha}\right)
$$

is the equilibrium distribution function for nearly incompressible fluids. M is an orthogonal transformation, which transforms the distribution functions from physical space into moment space [53], and $\hat{\mathbf{S}}$ is the diagonal relaxation matrix, which is chosen to be

$$
\hat{\mathbf{S}}=\operatorname{diag}\left(1,1,1,1,1,1,1, s_{\nu}, s_{\nu}\right)
$$


where

$$
s_{\nu}=\frac{1}{\tau+1 / 2}
$$

with $\tau$ being the hydrodynamic relaxation time (or simply the relaxation time), which is related to the kinematic viscosity of the system by $\nu=\tau c_{s}^{2} \delta t$, and is calculated by using a harmonic interpolation from the phase field

$$
\frac{1}{\tau}=\frac{1}{\tau_{\mathrm{L}}}+\phi\left(\frac{1}{\tau_{\mathrm{H}}}-\frac{1}{\tau_{\mathrm{L}}}\right)
$$

where $\tau_{\mathrm{L}}$ and $\tau_{\mathrm{H}}$ are the relaxation rates for the light and heavy fluids, respectively. If all the relaxation rates in Eq. (22) are set to $s_{\nu}$, the BGK model is recovered.

After solving LBE (17) using a routine collision-streaming sequence, hydrodynamic properties are calculated as

$$
\begin{aligned}
& \boldsymbol{u}=\frac{1}{\rho c_{s}^{2}} \sum_{\alpha} \bar{g}_{\alpha} \boldsymbol{e}_{\alpha}+\frac{\delta t}{2 \rho}\left(\boldsymbol{F}_{\mathrm{s}}+\boldsymbol{F}_{\mathrm{b}}\right) \\
& p=\sum_{\alpha} \bar{g}_{\alpha}+\frac{\delta t}{2}\left(\rho_{\mathrm{H}}-\rho_{\mathrm{L}}\right) c_{s}^{2} \boldsymbol{u} \cdot \boldsymbol{\nabla} \phi
\end{aligned}
$$

Note that velocity is updated before pressure.

It is worth mentioning that the only non-local macroscopic quantity in the proposed LB equations is the phase-field variable. Gradients of the phase field in Eq. (2) and Eq. (7), and its Laplacian in Eq. (8) are calculated using second-order, isotropic centered differences [54]. Moreover, we do not treat the $\boldsymbol{e}_{\alpha} \cdot \boldsymbol{\nabla} \phi$ term as a directional derivative along the lattice links [36], but instead compute the gradient of the phase field $\nabla \phi$ using isotropic differences and then execute the dot product, which improves the isotropy in the solution. We avoid using a combination of central and biased differences as was done in previous studies [36, 39], because it leads to violations of mass and 
momentum conservation [40]. Note that the main reason for using biased differences in Ref. [36] is to reduce the dispersion error in the numerical discretization of the advection term. Compared with the Cahn-Hilliard-based model in Ref. [36], the conservative phase-field LBE in Sec. (3.1) produces much less numerical dispersion, even when central differences are employed. This allows us to attain a stable numerical scheme at large density and viscosity ratios using a minimal number (three) of supporting nodes.

\section{Curved boundary treatment}

\subsection{Wetting boundary condition for the phase field}

In order to apply the wetting condition given in Eq. (4) on a curved boundary, we need the unit vector normal to the solid wall $\left(\hat{\boldsymbol{n}}_{\mathrm{w}}\right)$ along with the gradient of the phase field $\left.\boldsymbol{\nabla} \phi\right|_{\boldsymbol{x}_{\mathrm{w}}}$ and its value $\phi_{\mathrm{w}}$ at the wall. To this end we propose two different schemes.

A schematic implementation of the first approach for a typical boundary node is illustrated in Fig. 1. We use the fluid nodes, shown by blue dots, to obtain the unknown phase-field value at the boundary node $\left(\phi_{i, j}\right)$, shown by a black dot, using the following procedure: first, we invoke a centered difference for the left-hand-side of Eq. (4) to obtain

$$
\left.\hat{\boldsymbol{n}}_{\mathrm{w}} \cdot \boldsymbol{\nabla} \phi\right|_{\boldsymbol{x}_{\mathrm{w}}}=\left.\frac{\partial \phi}{\partial n_{\mathrm{w}}}\right|_{\boldsymbol{x}_{\mathrm{w}}}=\frac{\phi_{\mathrm{p}}-\phi_{i, j}}{2 h}=\Theta \phi_{\mathrm{w}}\left(1-\phi_{\mathrm{w}}\right)
$$

where $h=\left|\boldsymbol{x}_{\mathrm{p}}-\boldsymbol{x}_{\mathrm{w}}\right|$ is the distance between the solid wall and the interpolated point, which is known a priori given the location of the wall boundary. We eliminate $\phi_{\mathrm{w}}$ in Eq. (26) by using $\phi_{\mathrm{w}}=\left(\phi_{\mathrm{p}}+\phi_{i, j}\right) / 2$. This yields a 


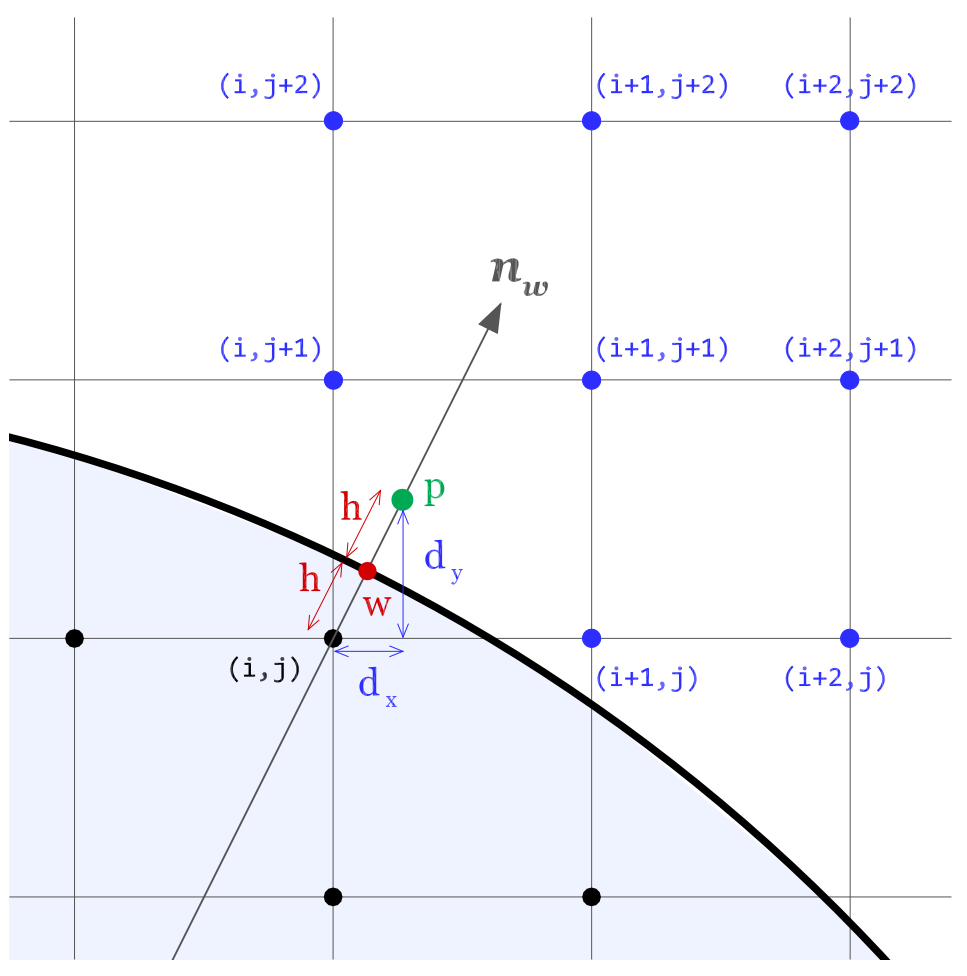

Figure 1: Schematic depicting the bidirectional interpolation to obtain the unknown phasefield value, $\phi_{i, j}$, at a typical boundary node (black dots). Blue dots are fluid nodes and $\boldsymbol{n}_{\mathrm{w}}$ is the normal vector pointing away from the solid wall. 
quadratic equation, the solution of which gives

$$
\phi_{i, j}=\frac{1}{a}\left(1+a-\sqrt{(1+a)^{2}-4 a \phi_{\mathrm{p}}}\right)-\phi_{\mathrm{p}}, \quad a=h \Theta \neq 0 \quad\left(\theta \neq 90^{\circ}\right)
$$

For neutral wetting conditions $\left(\theta=90^{\circ}\right)$, the trivial solution would be $\phi_{i, j}=$ $\phi_{\mathrm{p}}$. The only unknown quantity in Eq. (27) is $\phi_{\mathrm{p}}$, which can be determined by a biquadratic interpolation from the adjacent fluid nodes, as depicted in Fig. 1 . The only caveat is that $\phi_{i, j}$ will appear in the resulting bidirectional interpolation scheme. As a remedy, we can either solve Eq. (27) iteratively or use the value of $\phi_{i, j}$ from the previous time step. For slowly evolving flows, especially close to a stationary solid wall, $\phi_{i, j}$ is not expected to vary rapidly. As such, and also to avoid a costly iterative scheme, we choose the latter approach and use the value of $\phi_{i, j}$ from the previous time step in the bidirectional interpolation that is needed to find $\phi_{\mathrm{p}}$.

Alternatively, for our second approach, we can use unidirectional interpolations, either in the $x$-direction or in the $y$-direction, to find $\phi_{\mathrm{p}}$. A sketch demonstrating this approach is shown in Fig. 2. If the magnitude of the slope of the vector normal to the boundary is bigger than one, which is the case in Fig. 2(a), then the (linear or quadratic) interpolation is carried out in the $x$-direction; otherwise, the interpolation is carried out in the $y$-direction, as depicted in Fig. 2(b), to find the value of $\phi_{\mathrm{p}}$. Because the location of the solid wall $\boldsymbol{x}_{\mathrm{w}}$ is no longer half-way between the boundary node $\boldsymbol{x}_{i, j}$ and the interpolated point $\boldsymbol{x}_{\mathrm{p}}$, Eq. (26) must be modified. The final result is

$$
\phi_{i, j}=\frac{s+h}{2 a h}\left(1+a-\sqrt{(1+a)^{2}-4 a \phi_{\mathrm{p}}}\right)-\frac{s}{h} \phi_{\mathrm{p}}, \quad a=h \Theta \neq 0 \quad\left(\theta \neq 90^{\circ}\right)
$$

where $s=\left|\boldsymbol{x}_{\mathrm{w}}-\boldsymbol{x}_{i, j}\right|$ is the distance between the boundary node and the 


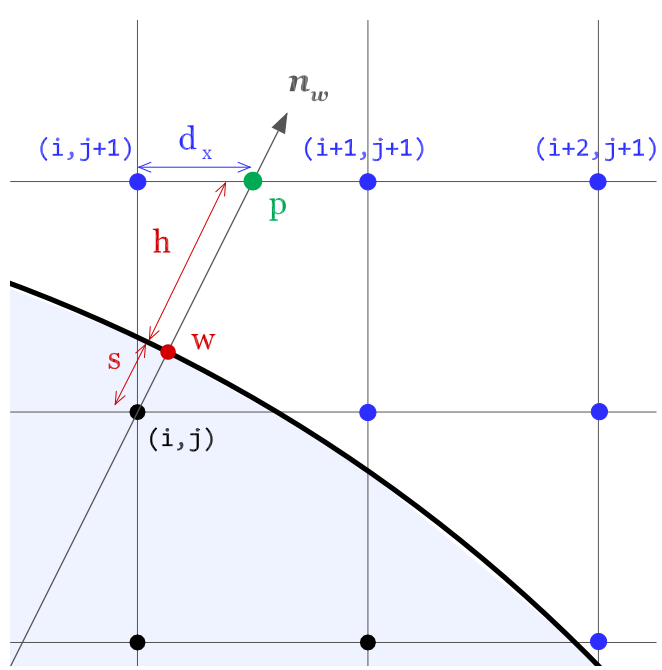

(a)

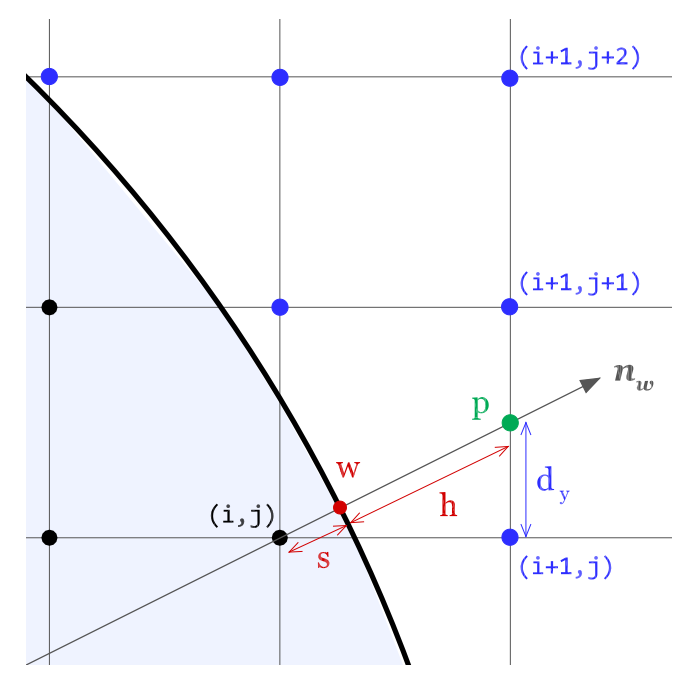

(b)

Figure 2: Unidirectional interpolation schemes. Schematic of boundary nodes and fluid nodes around a curve boundary.

solid wall.

\subsection{Boundary conditions for the distribution functions}

In addition to the wetting boundary condition for the phase field at the wall, the incoming distribution functions from the boundary nodes towards the fluid nodes must be determined. Referring to Fig. 3 for a typical boundary node $\boldsymbol{x}_{\mathrm{b}}$, and defining $\alpha^{-}$such that $\boldsymbol{e}_{\alpha^{-}}=-\boldsymbol{e}_{\alpha}$, the value of $\bar{g}_{\alpha^{-}}^{*}\left(\boldsymbol{x}_{\mathrm{b}}\right)$ before the streaming step is unknown (recall the asterisk denotes the pre-streaming, or post-collision, state). There are three common curved boundary models for specifying $\bar{g}_{\alpha^{-}}^{*}$ at the boundary:

\section{Filippova-Hänel model [55]:}

The boundary treatment of Filippova and Hänel [55], with the modifications proposed by Mei et al. [56], is a widely used curved boundary 


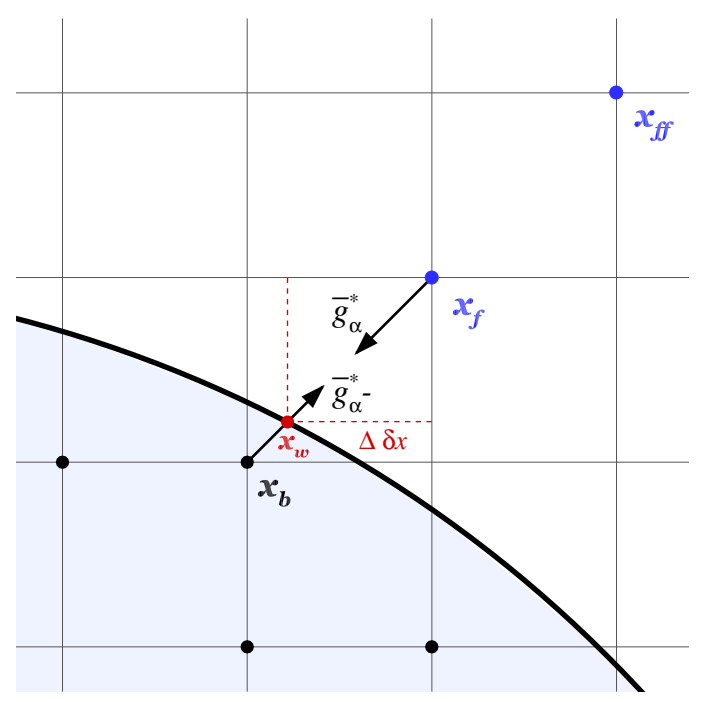

Figure 3: Schematic of boundary nodes and fluid nodes around a curved boundary.

273

274

condition in single-phase flows. Here, we use a variant of this model for a stationary solid boundary and a modified equilibrium distribution function to reduce the incompressibility error of the LBM. In essence, the following formula will be used to determine the unknown distribution functions at the solid boundaries

$$
\bar{g}_{\alpha^{-}}^{*}\left(\boldsymbol{x}_{\mathrm{b}}\right)=(1-\chi) \bar{g}_{\alpha}^{*}\left(\boldsymbol{x}_{\mathrm{f}}\right)+\chi \tilde{g}_{\alpha}\left(\boldsymbol{x}_{\mathrm{bf}}\right)
$$

where

$$
\tilde{g}_{\alpha}\left(\boldsymbol{x}_{\mathrm{bf}}\right)=g_{\alpha}^{e q}\left(\boldsymbol{x}_{\mathrm{f}}\right)+\rho\left(\boldsymbol{x}_{\mathrm{f}}\right) w_{\alpha} \boldsymbol{e}_{\alpha} \cdot\left(\boldsymbol{u}_{\mathrm{b}}-\boldsymbol{u}_{\mathrm{f}}\right)
$$

and

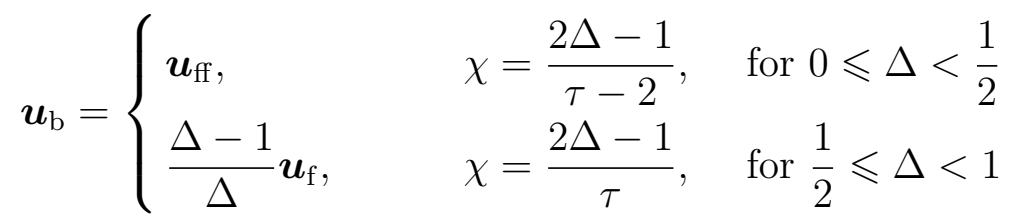


where

$$
\Delta=\frac{\left|\boldsymbol{x}_{\mathrm{f}}-\boldsymbol{x}_{\mathrm{w}}\right|}{\left|\boldsymbol{x}_{\mathrm{f}}-\boldsymbol{x}_{\mathrm{b}}\right|}
$$

2. Bouzidi-Firdaouss-Lallemand model [57]:

The following two-part formula was proposed by Bouzidi et al. [57]

$$
\bar{g}_{\alpha^{-}}^{*}\left(\boldsymbol{x}_{\mathrm{b}}\right)= \begin{cases}2 \Delta \bar{g}_{\alpha}^{*}\left(\boldsymbol{x}_{\mathrm{f}}\right)+(1-2 \Delta) \bar{g}_{\alpha}^{*}\left(\boldsymbol{x}_{\mathrm{ff}}\right), & 0 \leqslant \Delta<\frac{1}{2} \\ \frac{1}{2 \Delta} \bar{g}_{\alpha}^{*}\left(\boldsymbol{x}_{\mathrm{f}}\right)+\left(1-\frac{1}{2 \Delta}\right) \bar{g}_{\alpha^{-}}^{*}\left(\boldsymbol{x}_{\mathrm{ff}}\right), & \frac{1}{2} \leqslant \Delta<1\end{cases}
$$

\section{Yu-Mei-Shyy model [58]:}

$\mathrm{Yu}$ et al. [58] proposed a one-step relation to establish the incoming distribution functions according to

$$
\bar{g}_{\alpha^{-}}^{*}\left(\boldsymbol{x}_{\mathrm{b}}\right)=\frac{\Delta}{1+\Delta}\left[\bar{g}_{\alpha^{-}}^{*}\left(\boldsymbol{x}_{\mathrm{f}}\right)+\bar{g}_{\alpha}^{*}\left(\boldsymbol{x}_{\mathrm{f}}\right)\right]+\frac{1-\Delta}{1+\Delta} \bar{g}_{\alpha}^{*}\left(\boldsymbol{x}_{\mathrm{ff}}\right)
$$

It is worth noting that both Eq. (33) and (29) recover the commonly used mid-way bounce-back on the link [59] when $\Delta=1 / 2$, while the onestep formula (34) does not. Despite this, all the aforementioned methods produce virtually identical results in all of our simulations. We will only present the numerical results obtained using the first model [56].

In order to eliminate mass leakage from the wall boundaries, and also for stability reasons, we use mid-way bounce back to determine the unknown phase-field distribution function at the boundary nodes such that

$$
h_{\alpha^{-}}^{*}\left(\boldsymbol{x}_{\mathrm{b}}\right)=h_{\alpha}^{*}\left(\boldsymbol{x}_{\mathrm{f}}\right)
$$

Since we are dealing with stationary solid walls in this paper, the proposed curved boundary conditions can be applied very efficiently by storing 
the necessary data at the beginning of the simulation. Given a prespecified curved boundary we can identify the boundary nodes (shown by black dots in Figs. 1-3) and store their coordinate indices $(i, j)$ together with the necessary parameters $s, h, d_{x}, d_{y}$, and $\Delta$. For some problems (e.g. flow through complex porous media) the computations would be more efficient if the curved boundary treatment is turned off. We can easily achieve this while still using the proposed model by replacing curved boundaries with appropriate stair cases and implementing mid-way bounce-back. We can also deal with moving boundary problems by adding additional terms related to the motion of the boundary to the curved boundary models above. Here we only deal with stationary solid walls and leave the moving boundary problems to future studies.

\section{Numerical results}

In this section we test and validate the proposed LBM by considering various interesting problems involving three-phase contact line dynamics. We start simple with a drop on a flat wall, for which an analytical relation between the equilibrium contact angle and the height of the drop can be derived, and progressively add to the complexity of the problems. All input parameters will be presented in lattice units, or in dimensionless form, unless otherwise stated.

\subsection{Test 1: wetting/dewetting of a droplet on a flat surface}

As our initial condition we place a semi-circular droplet on top of a flat wall with an initial contact angle of $90^{\circ}$, which would be the equilibrium solution for neutral wetting conditions. We then specify different contact 


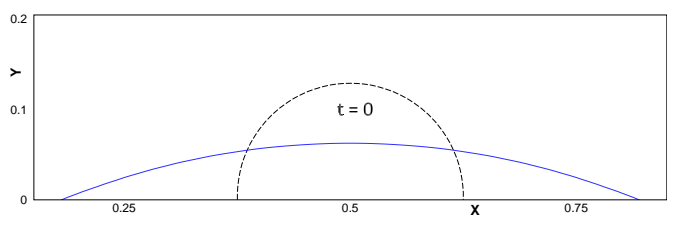

(a) $\theta=30^{\circ}$

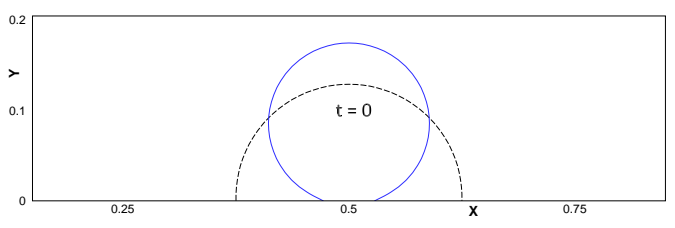

(b) $\theta=150^{\circ}$

Figure 4: Droplet wetting and dewetting on a (a) hydrophilic surface, and (b) hydrophobic surface.

angles by imposing boundary condition (4) and allow the droplet to evolve to its true equilibrium state. In the absence of gravity, the only mobilizing factor is interfacial tension. If $\theta<90^{\circ}$ the solid wall is said to be hydrophilic and the droplet starts wetting the surface spreading to increase its contact area with the surface until the equilibrium contact angle is attained. On the other hand, if $\theta>90^{\circ}$ then the solid wall is said to be hydrophobic and the droplet retracts in such a way as to reduce its contact area with the surface until the equilibrium contact angle is attained. Numerical results of these two states are shown in Fig. 4 for select contact angles. The simulation parameters are $\sigma=0.01, M=0.02, \rho_{\mathrm{H}} / \rho_{\mathrm{L}}=1000$, and $\mu_{\mathrm{H}} / \mu_{\mathrm{L}}=100$, where $\mu_{\mathrm{L}}$ and $\mu_{\mathrm{H}}$ are the bulk viscosities of the light and heavy fluids, respectively. The initial droplet is centered at $\left(L_{0} / 2,0\right)$, where $L_{0}$ is the length of the domain. The computational domain of size $L_{0} \times L_{0} / 2$ is resolved with $128 \times 64$ grid points. The initial radius of the semi-droplet is $R=L_{0} / 8=16$. Periodic boundary conditions are applied in the $x$-direction and mid-way bounce-back is used on the top boundary.

To test the accuracy of the results, we compare an analytical relationship between the specified contact angle $\theta$ and the maximum height of the droplet 


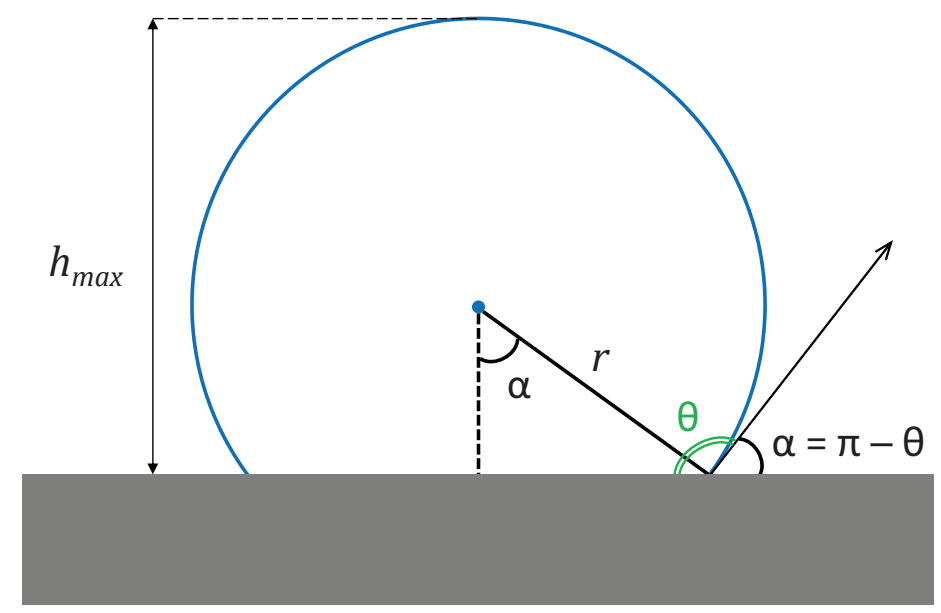

Figure 5: Contact angle $(\theta)$ between a droplet and a flat surface.

$h_{\text {max }}$ above the flat surface, as demonstrated in Fig. 5, at equilibrium for two different values of interfacial thickness, $\xi=3$ and $\xi=4$. The mass per unit density (area) of the droplet is $A^{e q}=r^{2}(\pi-\alpha+\sin \alpha \cos \alpha)$, where $\alpha=\pi-\theta$. Given an initial mass per unit density $A^{0}=\pi R^{2} / 2$, conservation of mass means

$$
\frac{h_{\max }}{R}=(1-\cos \theta) \sqrt{\frac{\pi}{2 \theta-\sin 2 \theta}}
$$

Figure 6 shows a good agreement between numerical results and analytical solution over a wide range of contact angles. The results for $\xi=4$ are slightly more accurate than those with $\xi=3$, particularly when the equilibrium contact angle is further away from $90^{\circ}$. The maximum error, which occurs for a hydrophilic surface with $\theta=30^{\circ}$, is measured at $16 \%$ for $\xi=3$ and $9 \%$ for $\xi=4$. In the rest of the simulations we use $\xi=4$ with $M=0.02$. 


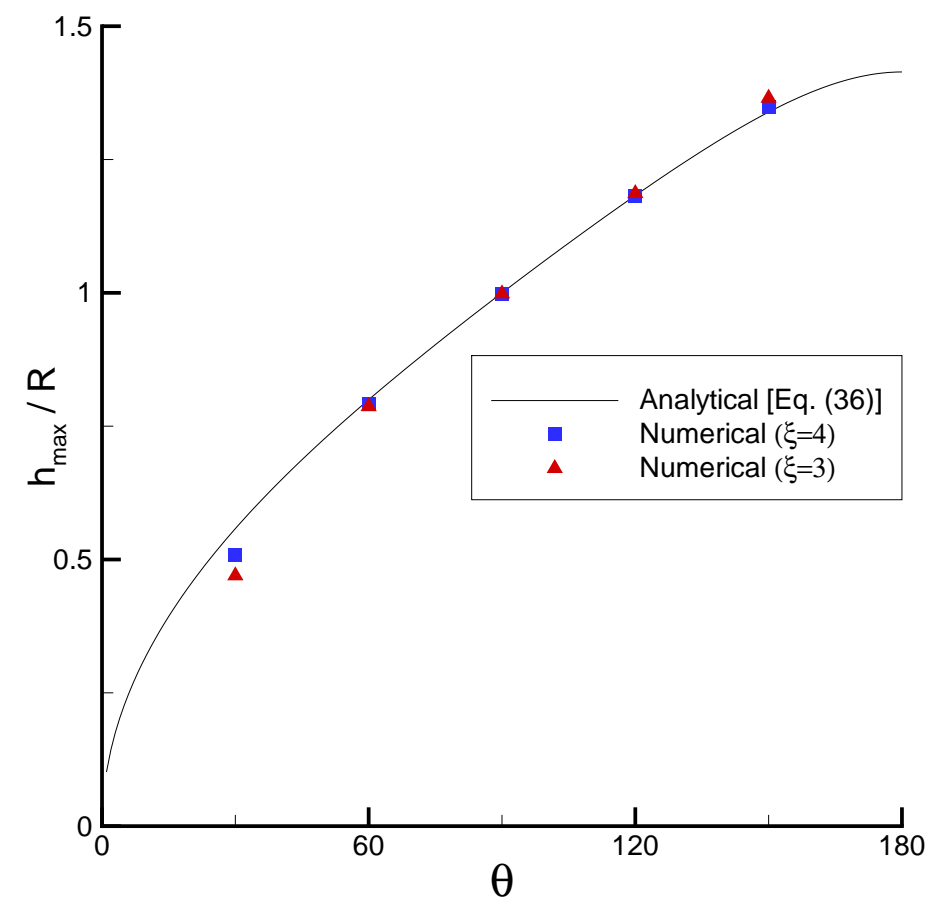

Figure 6: Contact angle versus dimensionless height of the drop on a flat surface. 


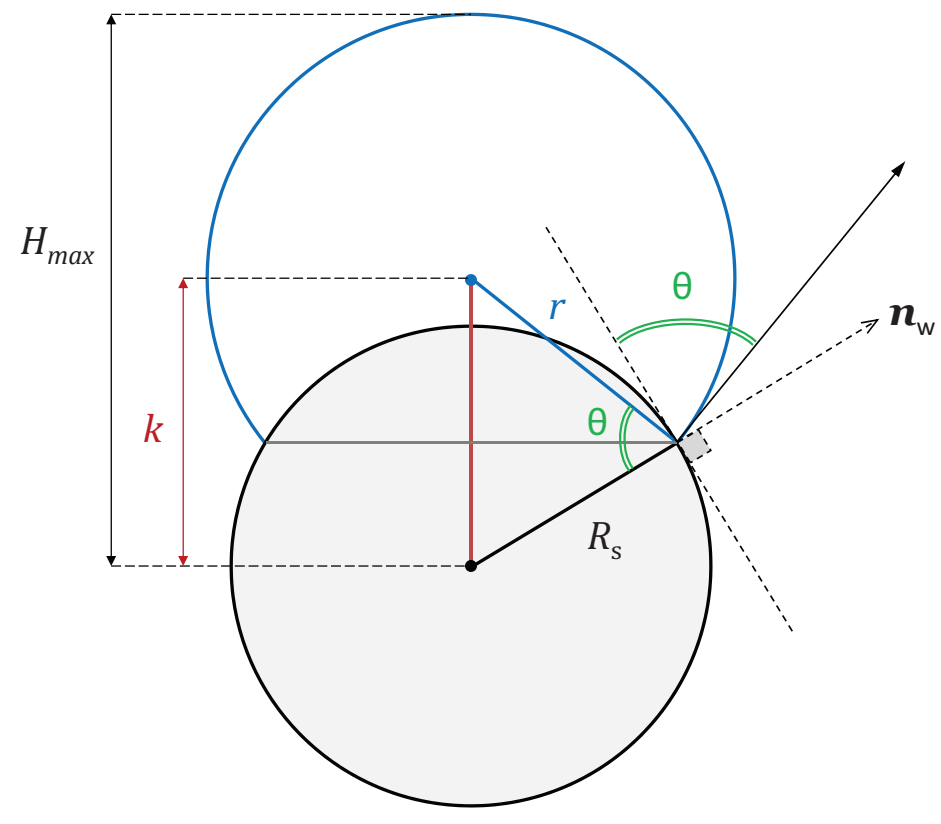

Figure 7: Schematic of the contact angle between a droplet with radius $r$ and a solid cylinder with radius $R_{\mathrm{s}}$. 


\subsection{Test 2: wetting/dewetting of a droplet on a cylindrical surface}

Next we will evaluate the accuracy of the proposed wetting boundary conditions on curved surfaces by placing a droplet on top of a circular cylinder in the absence of gravity. We specify contact angles ranging from $45^{\circ} \leq \theta \leq$ $135^{\circ}$. The droplet evolves until the prescribed contact angle at the threephase contact point is reached. A schematic of the shape of the droplet resting on the circular cylinder is shown in Fig. 7. At equilibrium, the interfacial tension forces tend to minimize the free energy in the system by minimizing the peripheral area. Therefore, an arc with radius $r$ is formed on top of the cylinder with radius $R_{\mathrm{s}}$. Referring to Fig. 7 , we can calculate the mass per unit density (area) of the circular droplet with radius $r$, whose center is distance $k$ from the center of the circular cylinder, in terms of $r$ and $k(r, \theta)$. The resulting relation is

$$
\begin{aligned}
A(r, k)=\pi r^{2} & -r^{2} \cos ^{-1}\left(\frac{k^{2}+r^{2}-R_{\mathrm{s}}^{2}}{2 k r}\right) \\
& -R_{\mathrm{s}}^{2} \cos ^{-1}\left(\frac{k^{2}-r^{2}+R_{\mathrm{s}}^{2}}{2 k R_{\mathrm{s}}}\right) \\
& +k \sqrt{R_{\mathrm{s}}^{2}-\left(\frac{k^{2}-r^{2}+R_{\mathrm{s}}^{2}}{2 k}\right)^{2}}
\end{aligned}
$$

where $k$ is related to $r$ and $\theta$ by the cosine law

$$
k(r, \theta)=\sqrt{r^{2}+R_{\mathrm{s}}^{2}-2 r R_{\mathrm{s}} \cos \theta} .
$$

We start the simulations by placing a circular cylinder centered at $\left(L_{0} / 2, L_{0} / 4\right)$ and a droplet with radius $R=R_{\mathrm{s}}=L_{0} / 6$ centered at $\left(L_{0} / 2, L_{0} / 4+R_{\mathrm{s}}\right)$. The properties of the fluids are $\rho_{\mathrm{H}} / \rho_{\mathrm{L}}=1000$ and $\mu_{\mathrm{H}} / \mu_{\mathrm{L}}=100$. First we examine the grid independency of the results on three different grids with 


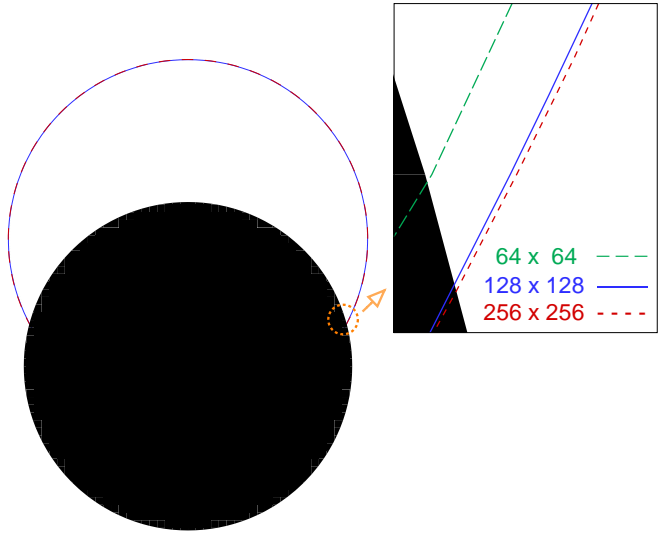

(a) $\theta=45^{\circ}$

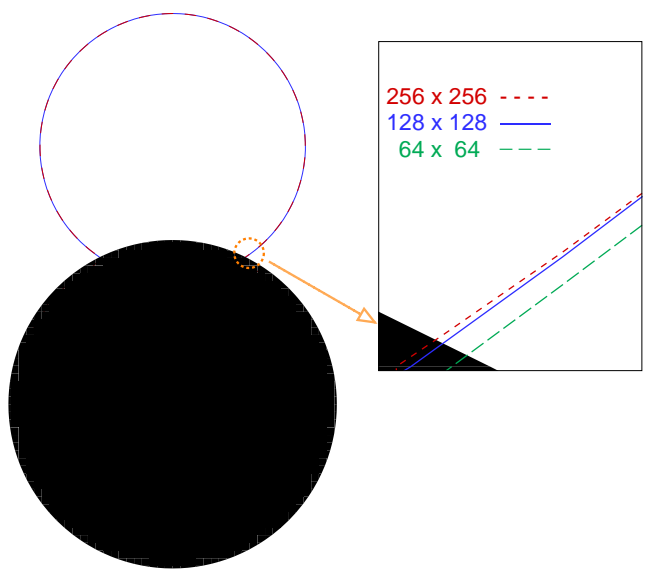

(b) $\theta=120^{\circ}$

Figure 8: Grid independency of the results for a droplet sitting on a cylindrical surface at $t \sigma / \mu_{\mathrm{H}} R=1800$. Comparison between three resolutions: $64 \times 64$ (dotted green line), $128 \times 128$ (solid blue line), and $256 \times 256$ (dashed red line).

$L_{0}=64,128$, and 256. Periodic boundary conditions are used in the $x$ direction and mid-way bounce-back is applied at the bottom and top of the domain. The results are shown in Fig. 8 for two different contact angles, corresponding to a hydrophilic $\left(\theta=45^{\circ}\right)$ and a hydrophobic $\left(\theta=120^{\circ}\right)$ surface, at $t \sigma / \mu_{\mathrm{H}} R=1800$. As can be observed, the drop interface is indistinguishable when increasing the number of grid points from $128 \times 128$ to $256 \times 256$. Therefore, we will use the $128 \times 128$ grid, with $\sigma=0.01$, to carry on the rest of the simulations in this section.

Figure 9 shows qualitative results for three different contact angles. Using $r=k=R_{\mathrm{s}}$ in Eq. (37) gives us the initial mass per unit density of the drop

$$
A\left(R_{\mathrm{s}}, R_{\mathrm{s}}\right)=\left(\frac{\pi}{3}+\frac{\sqrt{3}}{2}\right) R_{\mathrm{s}}^{2}
$$

By equating the initial mass of the drop in Eq. (39) with its equilibrium 


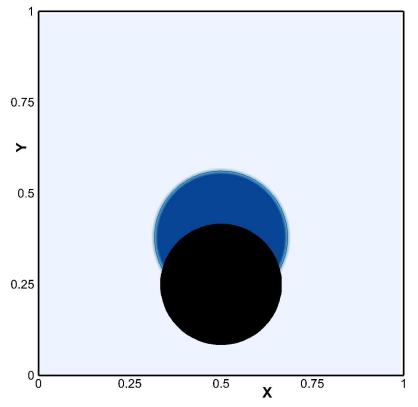

(a) $\theta=45^{\circ}$

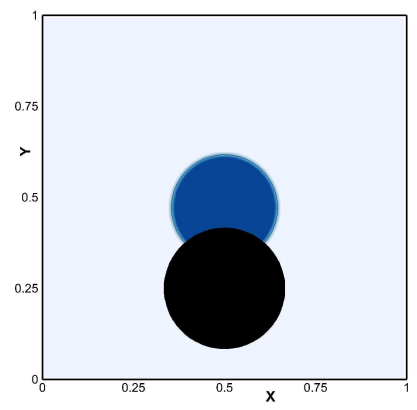

(b) $\theta=90^{\circ}$

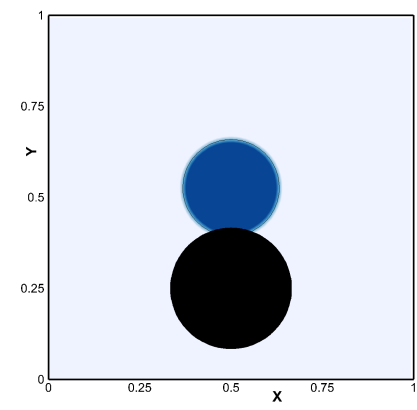

(c) $\theta=135^{\circ}$

Figure 9: Equilibrium profile of a droplet on a cylindrical surface for three different contact angles.

mass in Eq. (37) we obtain an implicit relation between $r$ and $\theta$. We solve the resulting relation iteratively using the secant method. We can then measure $H_{\max }$ (the distance between the top of the droplet and the center of the cylinder) in the simulations, and compare it with the analytical results $\left(H_{\max }=k+r\right)$. Variation of the dimensionless height of the drop $\left(H_{\max } / R\right)$ versus contact angle is plotted in Fig. 10. In addition to the numerical results obtained by the proposed curved boundary treatment, results obtained by approximating the curved wall as a stair-case (with bounce-back on the link), as is commonly done, are compared with the analytical solution. As can be seen in Fig. 10, approximating the curved wall with a stair-cased boundary leads to erroneous results, becoming progressively worse as the contact angle deviates more and more from neutral conditions. On the other hand, the numerical findings obtained by the present curved boundary model are in excellent agreement with the analytical solution. The maximum error, which occurs for a droplet in contact with a hydrophilic surface at $\theta=45^{\circ}$, is less than $1 \%$ for the curved boundary model while it is about $5 \%$ for the 
stair-cased approximation.

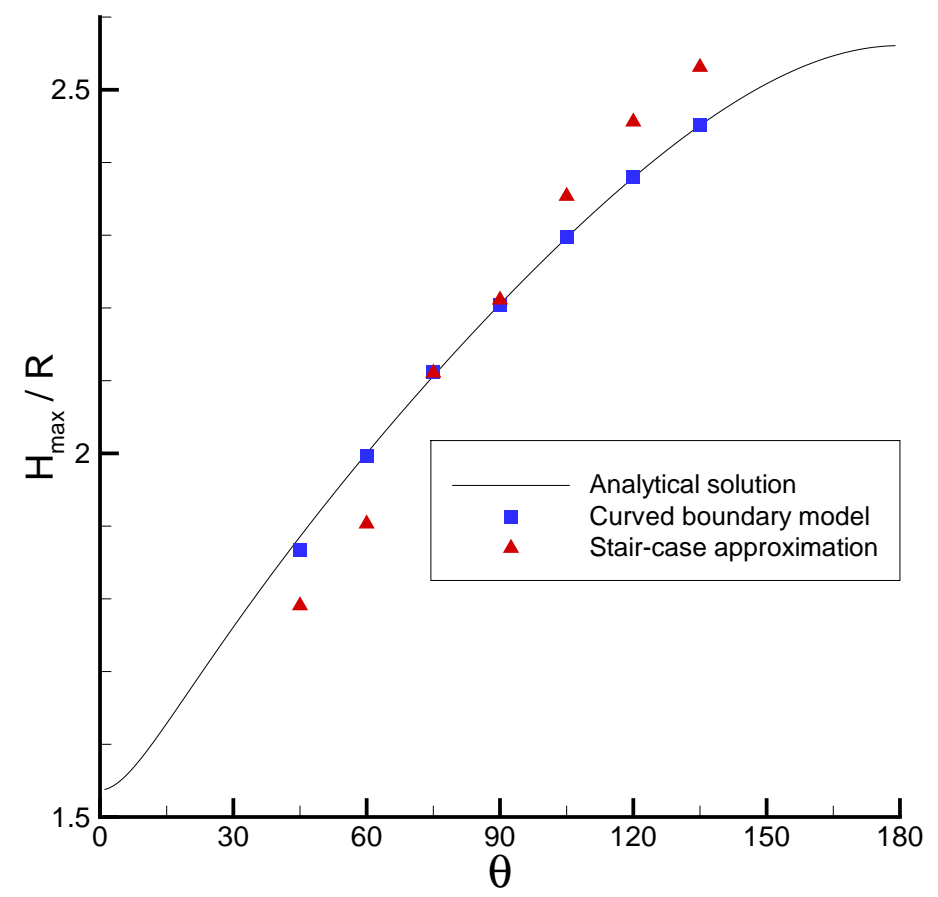

Figure 10: Comparison between the analytical solution and numerical results for a droplet in contact with a cylindrical surface.

\subsection{Test 3: unsteady flow past a circular cylinder}

Single-phase, incompressible, uniform flow past a circular cylinder is a standard and widely studied problem with very well documented results [6068]. Once the Reynolds number supersedes a threshold value, $\operatorname{Re}_{c r} \sim 49$ [63], the flow becomes unstable and the so-called von Kármán vortex street forms downstream of the cylinder. Here we extend this problem to a multiphase flow past a circular cylinder and compare the findings, particularly the drag and lift coefficients, with the available data for single-phase flow. 


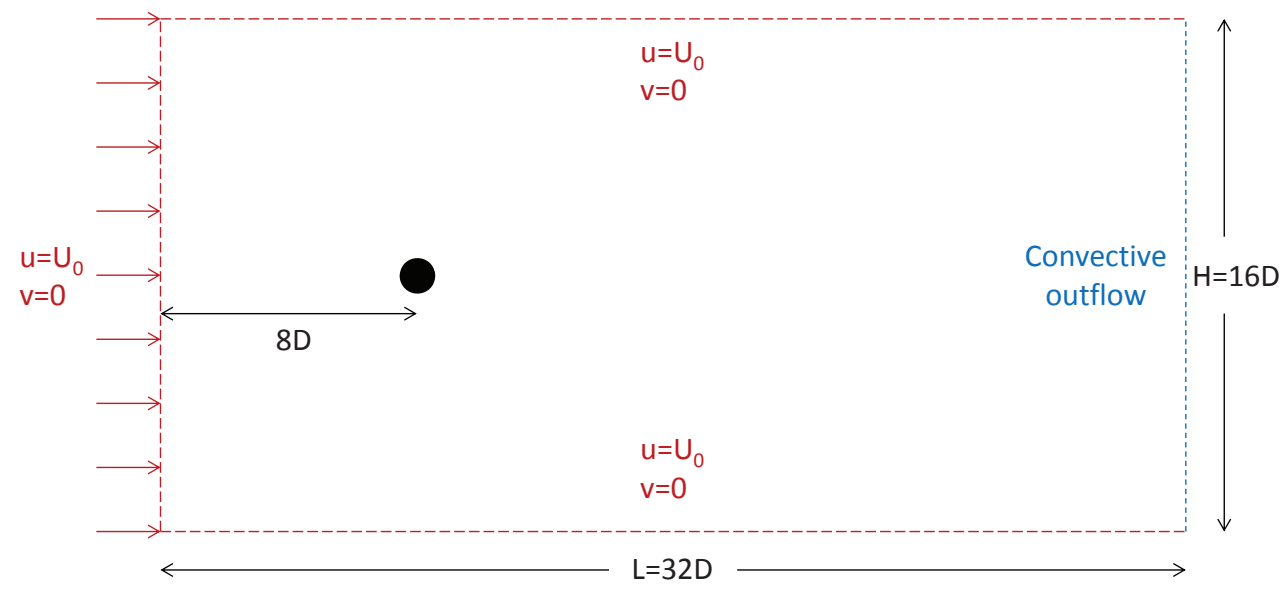

Figure 11: Schematic of the computational domain and boundary conditions for uniform flow past a circular cylinder.

The computational domain is shown in Fig. 11. A circular cylinder of diameter $D$ is centered at $x=8 D$ from the inlet. The length and height of the computational domain are $L=32 D$ and $H=16 D$, respectively. In order to expedite initiation of vortex shedding, the cylinder is displaced from the centerline by one lattice node in the $y$-direction. The no-slip boundary condition on the surface of the cylinder is realized by applying the boundary conditions in Eq. (29). A uniform flow $(u, v)=\left(U_{0}, 0\right)$ is imposed at the inlet and along the top and bottom of the domain by invoking the nonequilibrium bounce-back rule after the streaming step [69]

$$
f_{\alpha^{-}}=f_{\alpha}+f_{\alpha^{-}}^{e q}-f_{\alpha}^{e q}
$$

where $f_{\alpha} \in\left(h_{\alpha}, \bar{g}_{\alpha}\right)$ denotes the unknown incoming distribution function after the streaming step. For example, for the nearly incompressible LBM proposed by He and Luo [70], which is used in our single-phase flow simula- 
tions, the non-equilibrium bounce back at the inlet gives

$$
\bar{g}_{\alpha^{-}}=\bar{g}_{\alpha}+2 w_{\alpha} \rho_{0} U_{0}
$$

where $\alpha^{-} \in\{1,5,8\}, \alpha \in\{3,7,6\}$, and $\rho_{0}$ is a constant representing the density of the single-phase fluid. Convective boundary conditions for the macroscopic properties (such as $p, u, v$, etc.) are applied at the outlet by solving the following equation using an implicit, upwind finite-difference scheme:

$$
\frac{\partial q}{\partial t}+U_{0} \frac{\partial q}{\partial x}=0
$$

where $q$ is a placeholder for the macroscopic properties. Then the nonequilibrium bounce-back is invoked at the outlet to find the incoming distribution functions. The initial condition is the potential flow solution around a circular cylinder in an infinite domain. The Reynolds number of the flow $\operatorname{Re}=U_{0} D / \nu$ is fixed at $\operatorname{Re}=100$. The momentum-exchange method [71] is used to calculate the force exerted on the cylinder such that

$$
\boldsymbol{F}(t)=-\frac{1}{c_{s}^{2}} \sum_{\boldsymbol{x}_{\mathrm{b}}} \sum_{\alpha^{-}} \boldsymbol{e}_{\alpha^{-}}\left[\bar{g}_{\alpha^{-}}^{*}\left(\boldsymbol{x}_{\mathrm{b}}, t\right)+\bar{g}_{\alpha}^{*}\left(\boldsymbol{x}_{\mathrm{f}}, t\right)\right]
$$

where the summation at $\boldsymbol{x}=\boldsymbol{x}_{\mathrm{b}}$ is taken over the $\alpha^{-}$-directions that are pointing away from the circular cylinder. After calculating the force on the cylinder, the drag and lift coefficients are computed as

$$
\begin{aligned}
C_{\mathrm{D}} & =\frac{F_{x}}{1 / 2 \rho_{0} U_{0}^{2} D} \\
C_{\mathrm{L}} & =\frac{F_{y}}{1 / 2 \rho_{0} U_{0}^{2} D}
\end{aligned}
$$

Additionally we calculate the Strouhal number, defined as St $=f_{s} D / U_{0}$, where $f_{s}$ is the shedding frequency determined from the alternation of the lift force on the surface of the cylinder. 
Table 1: Average drag coefficient, peak-to-peak lift coefficient, and Strouhal number for unsteady flow past a circular cylinder at $\mathrm{Re}=100$.

\begin{tabular}{|c|c|c|c|}
\hline Reference & $\bar{C}_{\mathrm{D}}$ & $C_{\mathrm{L}}^{\text {peaks }}$ & St \\
\hline Liu et al. [66] & 1.350 & \pm 0.339 & 0.165 \\
Calhoun [67] & 1.330 & \pm 0.298 & 0.175 \\
present $\left(L_{0}=256\right)$ & 1.377 & \pm 0.324 & 0.170 \\
present $\left(L_{0}=512\right)$ & 1.400 & \pm 0.348 & 0.172 \\
present $\left(L_{0}=1024\right)$ & 1.408 & \pm 0.355 & 0.172 \\
\hline
\end{tabular}

\subsection{1. single-phase flow simulations}

First, the time-averaged drag coefficient is calculated on three different grids of differing resolution, which are $256 \times 128,512 \times 256$, and $1024 \times 512$, to examine grid independence of the results. The numerical findings, together with available experimental and numerical data, are provided in Table 1. As can be seen, the calculated drag coefficient and the Strouhal number are in reasonable agreement with the available data in the literature. Also, increasing the resolution from $512 \times 256$ to $1024 \times 512$ has minimal effect on the predicted values for the drag and Strouhal number. Therefore, the $512 \times 256$ grid will be used for further simulations. Variation of drag and lift coefficients versus dimensionless time $t^{*}=t U_{0} / D$ is also plotted in Fig. 12 .

\subsection{2. multiphase flow simulations}

Now we consider the dynamics of binary fluid flow past a circular cylinder. In order to study the effect of surface tension, the density ratio and the viscosity ratio are set to one and the wettability of the cylinder is examined 


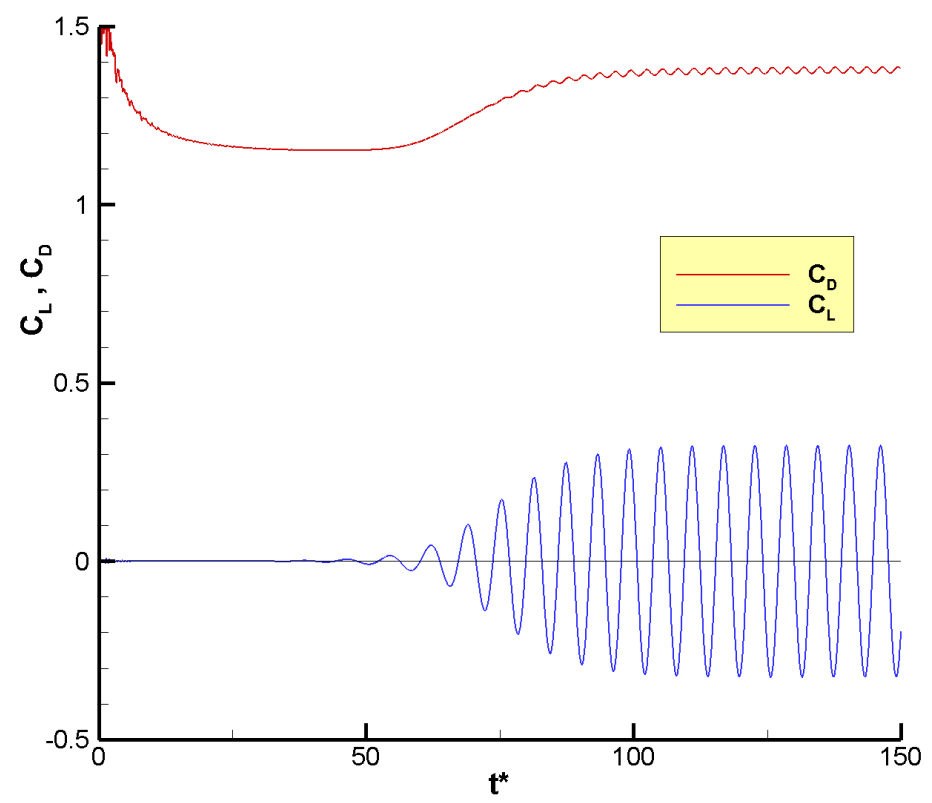

Figure 12: Variation of the drag and lift coefficients for the unsteady single-phase flow past a circular cylinder at $\operatorname{Re}=100$.

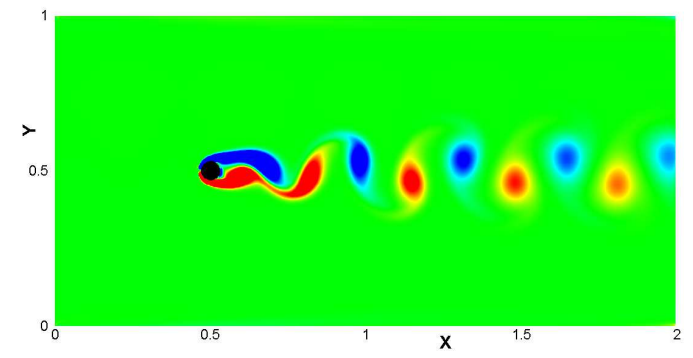

(a) single-phase

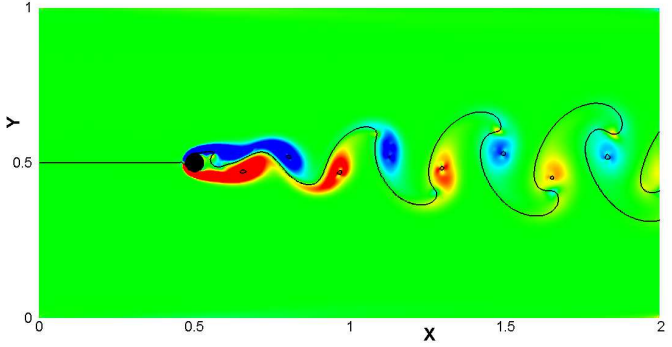

(b) multiphase $\left(\theta=30^{\circ}\right)$

Figure 13: Unsteady flow past a circular cylinder at $R e=100$. Left: vorticity (flooded contour) in the single-phase flow; Right: vorticity (flooded contour) and interface location (black line) in the multiphase flow. 


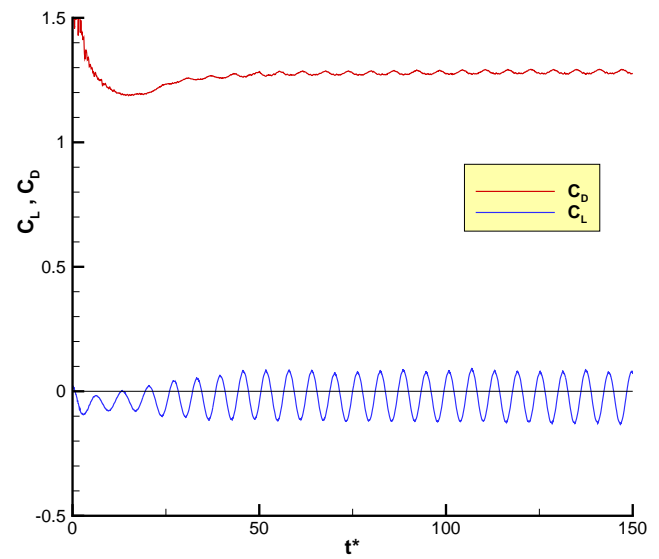

(a) $\theta=30^{\circ}$ contours in the multiphase flow simulations.

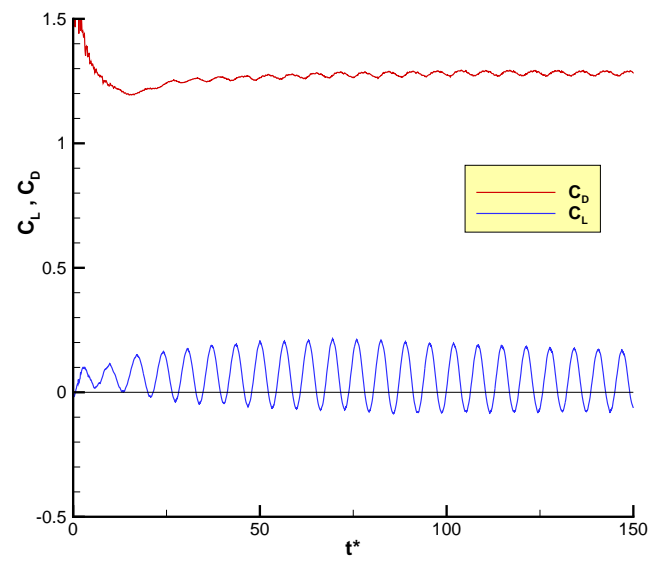

(b) $\theta=150^{\circ}$

Figure 14: Variation of the drag and lift coefficients for the unsteady multiphase flow past

a circular cylinder at $\mathrm{Re}=100$.

by changing the equilibrium contact angle. We consider two different contact angles: $\theta=30^{\circ}$ and $\theta=150^{\circ}$ with $\sigma=0.002$. Qualitative comparison between the single-phase flow and multiphase flow simulations is shown in Fig. 13. Although the vorticity contours for both cases are similar, the interfacial tension forces in the binary fluid case compete with viscous forces and cause more dissipation in the flow field as is evident from the faded vorticity

Similar to the single-phase flow simulations, we can monitor the variation of drag and lift coefficients versus time and measure the resulting Strouhal number. The drag and lift coefficients are plotted in Fig. 14 for two different contact angles. As can be seen, the averaged drag coefficient for both cylinders are almost identical and the values are lower than those in the single-phase flow case. Similar reductions in drag coefficient have been ob- 
Table 2: Average drag coefficient, peak-to-peak lift coefficient, and Strouhal number for multiphase flow past a circular cylinder at $\mathrm{Re}=100$.

\begin{tabular}{|c|c|c|c|c|}
\hline & $\bar{C}_{\mathrm{D}}$ & $C_{\mathrm{L}}^{\min }$ & $C_{\mathrm{L}}^{\max }$ & $\mathrm{St}$ \\
\hline$\theta=30^{\circ}$ & 1.284 & -0.131 & 0.078 & 0.159 \\
$\theta=150^{\circ}$ & 1.281 & -0.077 & 0.167 & 0.159 \\
\hline
\end{tabular}

served in simulations of viscoelastic polymeric flows around cylinder [72]. With regard to the lift, it is interesting to note that the average lift coefficient for both cases is non-zero. The circular cylinder with $\theta=150^{\circ}$ produces a slightly positive lift coefficient while the other cylinder with $\theta=30^{\circ}$ results in a slightly negative value for the average lift coefficient. The magnitude of the peak-to-peak lift coefficient or both cylinders are almost identical (due to antisymmetry in the equilibrium contact angles) but with opposite direction. The reason for this phenomenon becomes clear by referring to Fig. 15, which shows the equilibrium profile of a quiescent two-layer fluid in the vicinity of the cylinder for both cases. As can be inferred from Fig. 15(a), the fluids exert a negative (downward) lift force on the cylinder with $\theta=30^{\circ}$ while the net lift force experienced by the other cylinder with $\theta=150^{\circ}$ in Fig. 15(b) is positive (upwards). This is the reason we observe a nontrivial average lift coefficient in Fig. 14.

\subsection{Test 4: droplet falling on a circular cylinder}

In order to show the versatility of the proposed model in dealing with three-phase contact line dynamics on curved surfaces, we consider droplet impact dynamics on a circular cylinder with different wettability properties. 


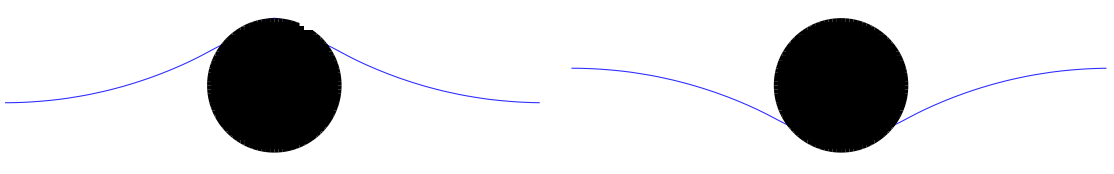
(a) $\theta=30^{\circ}$
(b) $\theta=150^{\circ}$

Figure 15: Equilibrium profile of a two-layer binary fluid around a solid cylinder for (a) $\theta=30^{\circ}$, and (b) $\theta=150^{\circ}$.

Droplet impact onto spherical and cylindrical objects has been investigated experimentally [73-76] and numerically [77, 42]. Most of these are for drop impact at large velocities (high Weber numbers). Our computational setup is depicted in Fig. 16. A circular droplet with radius $R=L_{0} / 10$ is centered at $\left(L_{0} / 2,3 L_{0} / 2\right)$ in a domain of size $L_{0} \times 2 L_{0}$, where $L_{0}=128$. A circular cylinder with radius $R_{\mathrm{s}}=R$ is also centered at $\left(L_{0} / 2, L_{0}\right)$. The droplet accelerates from rest towards the solid cylinder due to gravity, which is incorporated in the LBE via a body force $\boldsymbol{F}_{\mathrm{b}}=-\rho \mathrm{g}_{\mathrm{y}} \hat{\boldsymbol{j}}$, where $\mathrm{g}_{\mathrm{y}}$ is the magnitude of gravitational acceleration. The pressure field is initialized with a hydrostatic distribution. Periodic boundary conditions are applied in the $x$-direction and link bounce-back are applied at the bottom and top of the domain.

This problem is characterized by contact angle and four other dimensionless groups: density ratio $\rho_{\mathrm{H}} / \rho_{\mathrm{L}}$, viscosity ratio $\mu_{\mathrm{H}} / \mu_{\mathrm{L}}$, gravity Reynolds number $\operatorname{Re}_{\mathrm{Gr}}=\rho_{\mathrm{H}} \sqrt{\mathrm{g}_{\mathrm{y}} D^{3}} / \mu_{\mathrm{H}}$ and Eötvös or Bond number Bo $=\mathrm{g}_{\mathrm{y}}\left(\rho_{\mathrm{H}}-\rho_{\mathrm{L}}\right) D^{2} / \sigma$. Gravity-based dimensionless time is defined by $t_{g}^{*}=t \sqrt{\mathrm{g}_{\mathrm{y}} / D}$. In the literature, results are often also presented in terms of alternative dimension- 


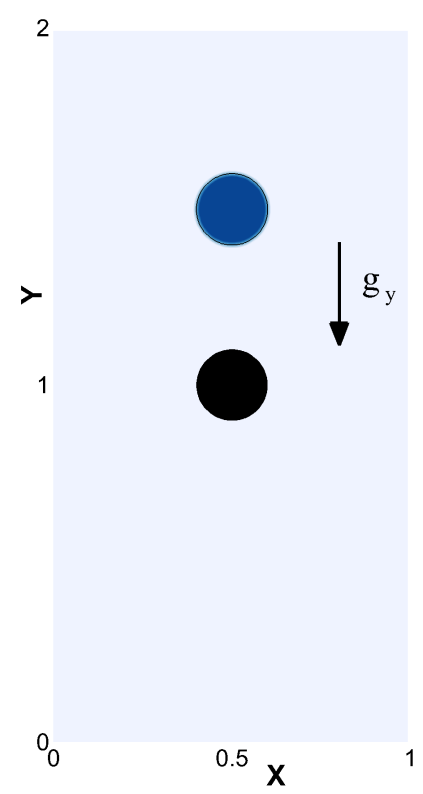

Figure 16: Computational domain for a droplet falling on a cylindrical surface.

less groups, the Reynolds and Weber numbers, where $\operatorname{Re}=\rho_{\mathrm{H}} U_{i} D / \mu_{\mathrm{H}}$ and We $=\rho_{\mathrm{H}} U_{i}^{2} D / \sigma$, respectively. These are based on the velocity of the droplet at the instant of impact, $U_{i}$. They can be combined to form the Ohnesorge number $\mathrm{Oh}=\sqrt{\mathrm{We}} / \mathrm{Re}$, which quantifies the ratio of viscous forces to surface tension forces. In all examples here we set the properties of the fluids such that $\rho_{\mathrm{H}} / \rho_{\mathrm{L}}=1000, \mu_{\mathrm{H}} / \mu_{\mathrm{L}}=100$, and $\operatorname{Re}_{\mathrm{Gr}}=25$. We then examine the effects of surface tension and contact angle at relatively small Bond numbers, Bo $\sim \mathcal{O}(1)$.

Results for a droplet impacting a hydrophilic surface with $\theta=40^{\circ}$ are shown in Fig. 17 for $\mathrm{Bo}=2.2(\mathrm{Oh}=0.06)$ and in Fig. 18 for $\mathrm{Bo}=6.6$ $(\mathrm{Oh}=0.11)$. For the lower Bond number in Fig. 17 the drop tends to adhere to the surface of the hydrophilic cylinder and evolve slowly. It then engulfs 


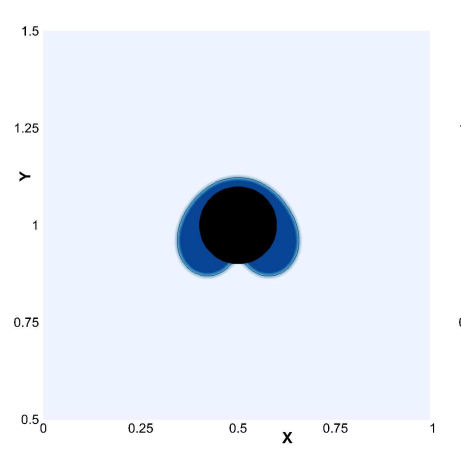

(a) $t_{g}^{*}=4.0$

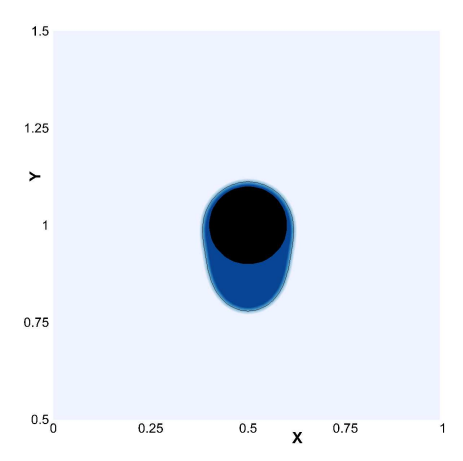

(b) $t_{g}^{*}=6.0$

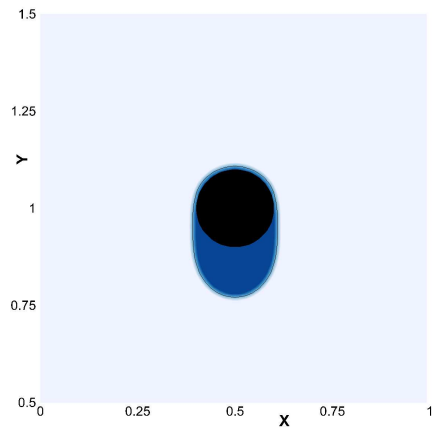

(c) $t_{g}^{*}=8.0$

Figure 17: Droplet impact on a hydrophilic cylindrical surface with $\theta=40^{\circ} \cdot \rho_{\mathrm{H}} / \rho_{\mathrm{L}}=1000$, $\mu_{\mathrm{H}} / \mu_{\mathrm{L}}=100, \mathrm{Re}_{\mathrm{Gr}}=25, \mathrm{Bo}=2.2(\mathrm{Oh}=0.06)$. See supplementary materials for simulation videos.

the perimeter of the hydrophilic cylinder at $t_{g}^{*}=6$ and starts stretching at $t_{g}^{*}=8$ due to the influence of gravity. External gravitational forces are not strong enough compared with the surface tension force between the droplet and the hydrophilic cylinder, causing the drop to momentarily hang from the circular surface while covering its whole area. On the other hand, the gravitational force in Fig. 18 is strong enough to prevail over the interfacial tension between the drop and the solid surface, causing the droplet to break up and detach from the surface of the hydrophilic cylinder.

In order to consider wettability effects of the cylindrical surface we increase the contact angle to $\theta=170^{\circ}$, which corresponds to a superhydrophobic surface. Dealing with a superhydrophobic surface numerically is rather challenging and its simulation poses interesting interfacial dynamics. As such, we consider droplet impact on the superhydrophobic cylinder for three different Bond numbers. Results are shown in Fig. 19 for $\mathrm{Bo}=2.2(\mathrm{Oh}=0.06)$, in 


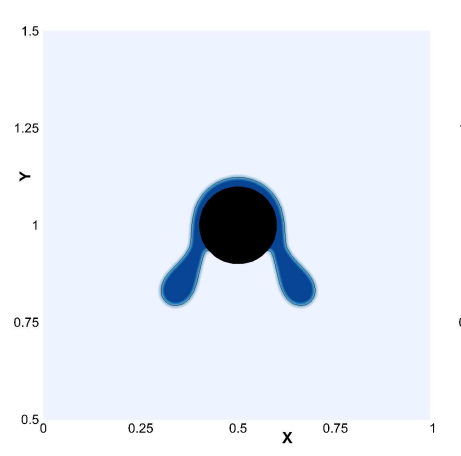

(a) $t_{g}^{*}=3.2$

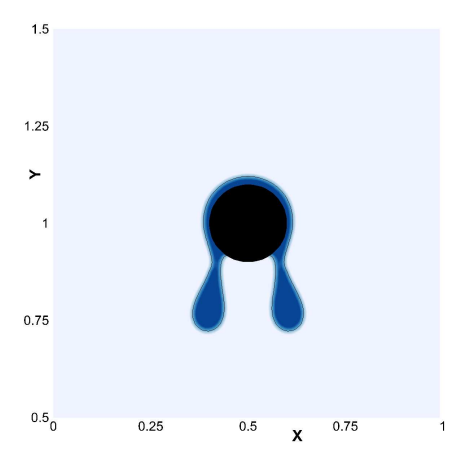

(b) $t_{g}^{*}=3.6$

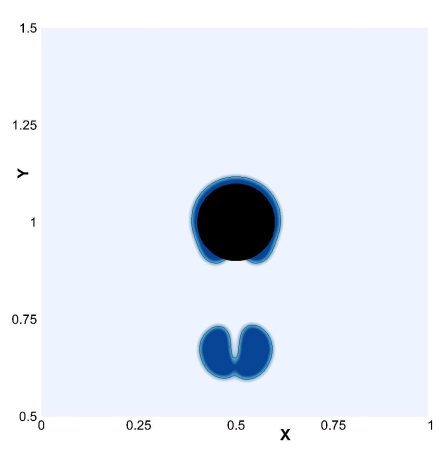

(c) $t_{g}^{*}=4.4$

Figure 18: Droplet impact on a hydrophilic cylindrical surface with $\theta=40^{\circ} \cdot \rho_{\mathrm{H}} / \rho_{\mathrm{L}}=1000$, $\mu_{\mathrm{H}} / \mu_{\mathrm{L}}=100, \mathrm{Re}_{\mathrm{Gr}}=25, \mathrm{Bo}=6.6(\mathrm{Oh}=0.11)$. See supplementary materials for simulation videos.

Fig. 20 for $\mathrm{Bo}=3.3(\mathrm{Oh}=0.07)$, and in Fig. 21 for $\mathrm{Bo}=6.6(\mathrm{Oh}=0.11)$.
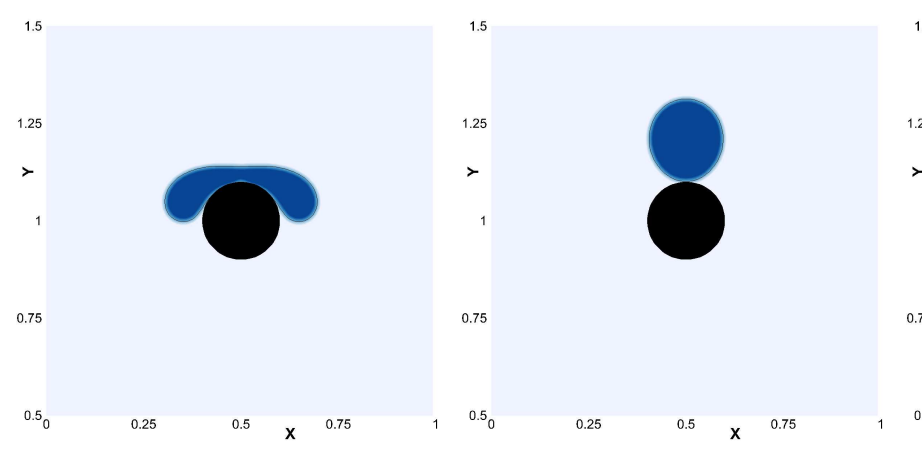
(a) $t_{g}^{*}=2.8$
(b) $t_{g}^{*}=4.8$
(c) $t_{g}^{*}=8.0$

Figure 19: Droplet impact on a superhydrophobic cylinder with $\theta=170^{\circ} \cdot \rho_{\mathrm{H}} / \rho_{\mathrm{L}}=1000$, $\mu_{\mathrm{H}} / \mu_{\mathrm{L}}=100, \operatorname{Re}_{\mathrm{Gr}}=25, \mathrm{Bo}=2.2(\mathrm{Oh}=0.06)$. See supplementary materials for simulation videos.

For the lowest Bond number in Fig. 19, the droplet retracts after hitting the cylindrical surface. The superhydrophobicity of the cylinder clearly overcomes the gravitational pulling force, causing the droplet to bounce on top of 


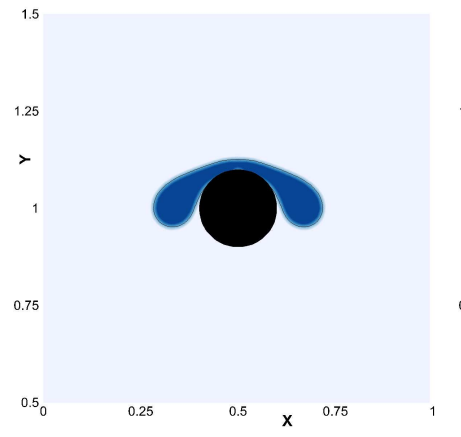

(a) $t_{g}^{*}=2.8$

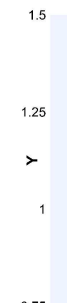

0.75
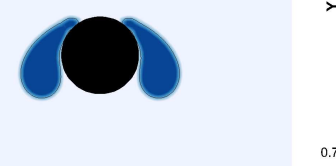

0.25

(b) $t_{g}^{*}=3.6$

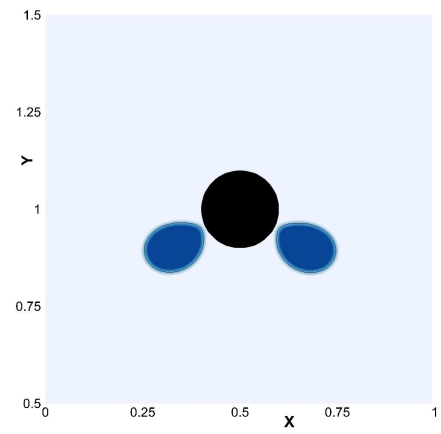

(c) $t_{g}^{*}=4.4$

Figure 20: Droplet impact on a superhydrophobic cylinder with $\theta=170^{\circ} \cdot \rho_{\mathrm{H}} / \rho_{\mathrm{L}}=1000$, $\mu_{\mathrm{H}} / \mu_{\mathrm{L}}=100, \mathrm{Re}_{\mathrm{Gr}}=25, \mathrm{Bo}=3.3(\mathrm{Oh}=0.07)$. See supplementary materials for simulation videos.

Comparing Fig. 19 and Fig. 17 at $t_{g}^{*}=8$, we can see the effect of wettability of the cylindrical surface on the outcome of droplet impact. The hydrophilic surface in Fig. 17 allows the drop to wet its entire perimeter while the superhydrophobic surface in Fig. 19 repels the drop. By increasing the Bond number to 3.3 in Fig. 20, the drop gains enough momentum to break up upon contact with the upper pole of the cylinder, while being repelled from the superhydrophobic surface. Eventually, the drop disintegrates into two daughter droplets which are completely detached from the surface of the cylinder at $t_{g}^{*}=4.4$, experiencing a free fall. Further increasing the Bond number to 6.6 leads to an interesting disintegration of the impacting drop 
into several droplets of various sizes in Fig. 21. Interested readers can refer to supplementary materials for simulation videos of these droplet impacts on the cylindrical surface.

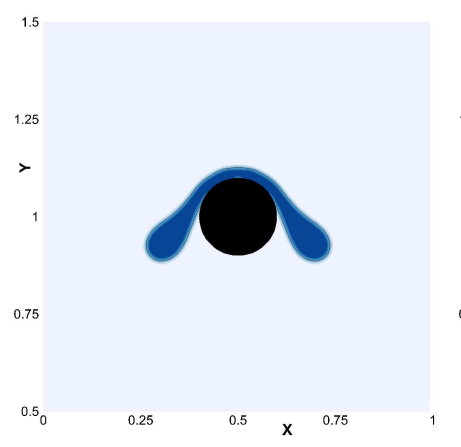

(a) $t_{g}^{*}=2.8$

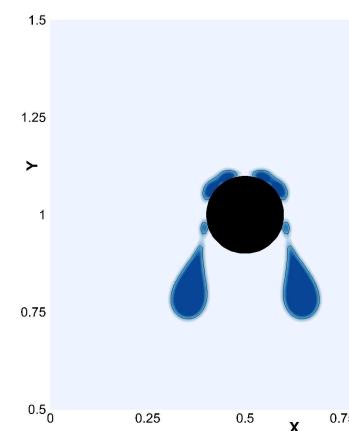

(b) $t_{g}^{*}=3.6$

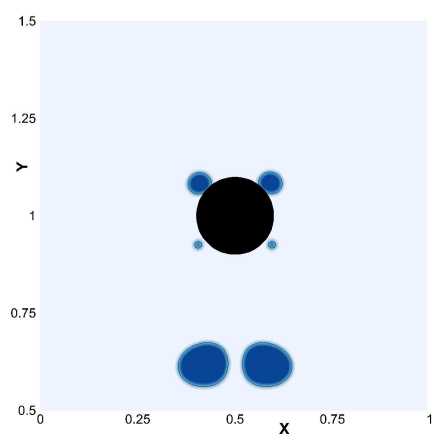

(c) $t_{g}^{*}=4.4$

Figure 21: Droplet impact on a superhydrophobic cylinder with $\theta=170^{\circ} \cdot \rho_{\mathrm{H}} / \rho_{\mathrm{L}}=1000$, $\mu_{\mathrm{H}} / \mu_{\mathrm{L}}=100, \operatorname{Re}_{\mathrm{Gr}}=25, \mathrm{Bo}=6.6(\mathrm{Oh}=0.11)$. See supplementary materials for simulation videos. 75]. According to [75] the film thickness is expected to vary as $h^{*}=1-t^{*}$ at early times, and as $h^{*}=0.15 t^{*-2}$ at intermediate times [75]. As can be seen in Fig. 22, our simulations approximately obey the power-law fitted curves of [75]. Any deviations are likely due to the unrealistic nature of our $2 \mathrm{D}$ 
simulations as well as the lower impact velocities in the current simulations compared with the experiments [75], which are conducted for a drop impact on a spherical surface with a drop diameter to target diameter ratio of 2.6/3.2 at $\operatorname{Re}=4806$ and $\mathrm{We}=131$. Despite these obvious limitations, our results are qualitatively promising.

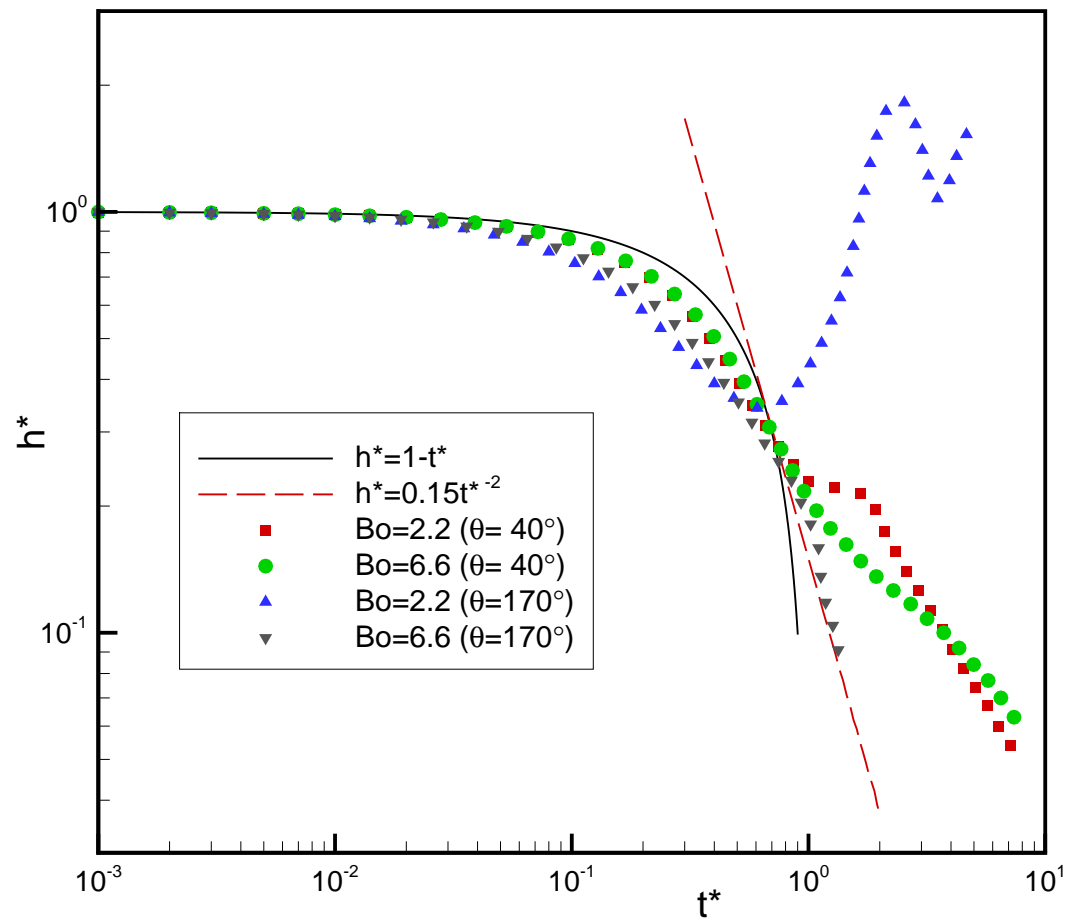

Figure 22: Temporal variation of the film thickness on top of the solid cylinder. $\rho_{\mathrm{H}} / \rho_{\mathrm{L}}=$ $1000, \mu_{\mathrm{H}} / \mu_{\mathrm{L}}=100$, and $\mathrm{Re}_{\mathrm{Gr}}=25$. Solid black line and dashed red line are the powerlaw curves from experimental data [75]; red squares correspond to $\theta=40^{\circ}$ and $\mathrm{Bo}=2.2$ $(\operatorname{Re}=30$ and $\mathrm{We}=3)$; green circles correspond to $\theta=40^{\circ}$ and $\mathrm{Bo}=6.6(\mathrm{Re}=32$ and $\mathrm{We}=12)$; blue triangles correspond to $\theta=170^{\circ}$ and $\mathrm{Bo}=2.2(\mathrm{Re}=21$ and $\mathrm{We}=2)$; and gray deltas correspond to $\theta=170^{\circ}$ and $\mathrm{Bo}=6.6(\mathrm{Re}=26$ and $\mathrm{We}=7)$. 


\section{Summary and conclusion}

In this paper we proposed a numerical technique to implement wetting boundary conditions on curved surfaces without approximating the curved boundary as a stair-case. We introduced a rather simple lattice Boltzmann method, implemented on uniform Cartesian grids, which is capable of simulating multiphase flows with large density ratios. In contrast to other existing LB models for multiphase flows, there is only one nonlocal macroscopic property in our model - the phase field. This feature is advantageous for parallel implementation of the algorithm. We conducted various benchmark studies, looking at three-phase contact line dynamics, to assess the proposed model. Where available, the numerical results were found to be in good agreement with analytical solutions and experimental data. The successful simulation of drop impingement on a superhydrophobic cylinder highlights the great potential of the proposed model for multiscale study of contact line dynamics within diffuse-interface models. Although some major studies have addressed the sharp-interface limit of Cahn-Hilliard-based models [9, 79], there is little to no work on this issue for conservative phase-field models [11], particularly when contact line motion is considered. Moreover, parameterizing the interplay between the mobility and interfacial thickness is another important topic worthy of investigation for accurate modeling of multiphase flows using the phase-field models. Both these issues present important future research avenues. 


\section{Acknowledgment}

AF would like to thank insightful discussions with Travis Mitchell. This material is based upon work supported, in part, by NSF grants EAR-1351625 and EAR-1417264. Any opinions, findings, conclusions, or recommendations do not necessarily reflect the views of the funding agencies.

\section{References}

[1] J. F. Morris, Handbook of Granular Materials, CRC Press, p. 391.

[2] Y. D. Shikhmurzaev, Capillary Flows with Forming Interfaces, Chapman and Hall/CRC, 2008.

[3] A. Prosperetti, G. Tryggvason, Computational Methods for Multiphase Flow, Cambridge University Press, 2009.

[4] J. S. Rowlinson, B. Widom, Molecular Theory of Capillarity, Clarendon Press, Oxford, 1989.

[5] D. Jacqmin, Calculation of two-phase Navier-Stokes flows using phasefield modeling, J. Comput. Phys. 155 (1999) 96.

[6] D. Jacqmin, Contact-line dynamics of a diffuse fluid interface, J. Fluid Mech. 402 (2000) 57-88.

[7] D. Jamet, O. Lebaigue, N. Coutris, J. Delhaye, The second gradient method for the direct numerical simulation of liquid-vapor flows with phase change, J. Comput. Phys. 169 (2001) 624. 
[8] L. M. Pismen, Nonlocal diffuse interface theory of thin films and the moving contact line, Phys. Rev. E 64 (2001) 021603.

[9] F. Magaletti, F. Picano, M. Chinappi, L. Marino, C. M. Casciola, The sharp-interface limit of the Cahn-Hilliard/Navier-Stokes model for binary fluids, J. Fluid Mech. 714 (2013) 95-126.

[10] J. W. Cahn, J. E. Hilliard, Free energy of a nonuniform system. I. Interfacial free energy, J. Chem. Phys. 28 (1958) 258.

[11] Y. Sun, C. Beckermann, Sharp interface tracking using the phase-field equation, J. Comput. Phys. 220 (2007) 626-653.

[12] P.-H. Chiu, Y.-T. Lin, A conservative phase field method for solving incompressible two-phase flows, J. Comput. Phys. 230 (2011) 185-204.

[13] H. Ding, P. D. M. Spelt, C. Shu, Diffuse interface model for incompressible two-phase flows with large density ratios, J. Comput. Phys. 226 (2007) 2078.

[14] X. He, L.-S. Luo, A priori derivation of the lattice Boltzmann equation, Phys. Rev. E 55 (1997) R6333-R6336.

[15] X. He, L.-S. Luo, Theory of the lattice Boltzmann method: From the Boltzmann equation to the lattice Boltzmann equation, Phys. Rev. E 56 (1997) 6811.

[16] X. He, G. D. Doolen, Thermodynamic foundations of kinetic theory and lattice Boltzmann models for multiphase flows, J. Stat. Phys. 107 (2002) 309 . 
[17] D. Yu, R. Mei, L.-S. Luo, W. Shyy, Viscous flow computations with the method of lattice Boltzmann equation, Prog. Aerosp. Sci. 39 (2003) 329.

[18] M. C. Sukop, D. T. Thorne, Lattice Boltzmann Modeling: An Introduction for Geoscientists and Engineers, Springer, Berlin, 2006.

[19] H. Huang, M. C. Sukop, X.-Y. Lu, Multiphase Lattice Boltzmann Methods: Theory and Application, Wiley-Blackwell, 2015.

[20] A. K. Gunstensen, D. H. Rothman, S. Zaleski, G. Zanetti, Lattice Boltzmann model of immiscible fluids, Phys. Rev. A 43 (1991) 4320.

[21] D. Grunau, S. Chen, K. Eggert, A lattice Boltzmann model for multiphase fluid flows, Phys. Fluids A 5 (1993) 2557.

[22] X. Shan, H. Chen, Lattice Boltzmann model for simulating flows with multiple phases and components, Phys. Rev. E 47 (1993) 1815.

[23] X. Shan, H. Chen, Simulation of nonideal gases and liquid-gas phase transitions by the latice Boltzmann equation, Phys. Rev. E 49 (1994) 2941.

[24] X. Shan, G. D. Doolen, Multicomponent lattice-Boltzmann model with interparticle interaction, J. Stat. Phys. 81 (1995) 379.

[25] X. Shan, G. Doolen, Diffusion in a multicomponent lattice Boltzmann equation model, Phys. Rev. E 54 (1996) 3614.

[26] M. R. Swift, W. R. Osborn, J. M. Yeomans, Lattice Boltzmann simulation of nonideal fluids, Phys. Rev. Lett. 75 (1995) 830. 
[27] E. Orlandini, M. R. Swift, J. M. Yeomans, A lattice Boltzmann model of binary-fluid mixtures, Europhys. Lett. 32 (1995) 463.

[28] M. R. Swift, E. Orlandini, W. R. Osborn, J. M. Yeomans, Lattice Boltzmann simulations of liquid-gas and binary fluid systems, Phys. Rev. E 54 (1996) 5041.

[29] X. He, X. Shan, G. D. Doolen, A discrete Boltzmann equation model for non-ideal gases, Phys. Rev. E 57 (1998) R13.

[30] X. He, S. Chen, R. Zhang, A lattice Boltzmann scheme for incompressible multiphase flow and its application in simulation of Rayleigh-Taylor instability, J. Comput. Phys. 152 (1999) 642.

[31] T. Inamuro, T. Ogata, S. Tajima, N. Konishi, A lattice Boltzmann method for incompressible two-phase flows with large density differences, J. Comput. Phys. 198 (2004) 628.

[32] T. Lee, C.-L. Lin, A stable discretization of the lattice Boltzmann equation for simulation of incompressible two-phase flows at high density ratio, J. Comput. Phys. 206 (2005) 16.

[33] Q. Li, K. H. Luo, X. J. Li, Lattice boltzmann modeling of multiphase flows at large density ratio with an improved pseudopotential model, Phys. Rev. E 87 (2013) 053301.

[34] S. Leclaire, K. Abahri, R. Belarbi, R. Bennacer, Modeling of static contact angles with curved boundaries using a multiphase lattice Boltzmann method with variable density and viscosity ratios, 2016. Fld.4226. 
[35] D. Chiappini, G. Bella, S. Succi, F. Toschi, S. Ubertini, Improved lattice Boltzmann without parasitic currents for Rayleigh-Taylor instability, Commun. Comput. Phys. 7 (2010) 423-444.

[36] T. Lee, L. Liu, Lattice Boltzmann simulations of micron-scale drop impact on dry surfaces, J. Comput. Phys. 229 (2010) 8045.

[37] H. W. Zheng, C. Shu, Y. T. Chew, A lattice Boltzmann model for multiphase flows with large density ratio, J. Comput. Phys. 218 (2006) 353.

[38] A. Fakhari, M. H. Rahimian, Phase-field modeling by the method of lattice Boltzmann equations, Phys. Rev. E 81 (2010) 036707.

[39] A. Fakhari, T. Lee, Multiple-relaxation-time lattice Boltzmann method for immiscible fluids at high Reynolds numbers, Phys. Rev. E 87 (2013) 023304.

[40] Z. Guo, C. Zheng, B. Shi, Force imbalance in lattice Boltzmann equation for two-phase flows, Phys. Rev. E 83 (2011) 036707.

[41] A. Fakhari, M. Geier, T. Lee, A mass-conserving lattice Boltzmann method with dynamic grid refinement for immiscible two-phase flows, J. Comput. Phys. 315 (2016) 434-457.

[42] H.-R. Liu, H. Ding, A diffuse-interface immersed-boundary method for two-dimensional simulation of flows with moving contact lines on curved substrates, J. Comput. Phys. 294 (2015) 484-502. 
[43] H. Liu, A. J. Valocchi, Q. Kang, Three-dimensional lattice Boltzmann model for immiscible two-phase flow simulations, Phys. Rev. E 85 (2012) 046309 .

[44] Y. Ba, H. Liu, Q. Li, Q. Kang, J. Sun, Multiple-relaxation-time colorgradient lattice Boltzmann model for simulating two-phase flows with high density ratio, Phys. Rev. E 94 (2016) 023310.

[45] S. Leclaire, N. Pellerin, M. Reggio, J. Trépanier, An approach to control the spurious currents in a multiphase lattice Boltzmann method and to improve the implementation of initial condition, Int. J. Numer. Methods Fluids 77 (2015) 732-746.

[46] S. Leclaire, N. Pellerin, M. Reggio, J. Trépanier, A multiphase lattice Boltzmann method for simulating immiscible liquid-liquid interface dynamics, Appl. Math. Model. 40 (2016) 6376-6394.

[47] H. Liu, Q. Kang, C. R. Leonardi, S. Schmieschek, A. Narváez, B. D. Jones, J. R. Williams, A. J. Valocchi, J. Harting, Multiphase lattice Boltzmann simulations for porous media applications, Comput. Geosci. 20 (2016) 777-805.

[48] A. Fakhari, M. H. Rahimian, Investigation of deformation and breakup of a falling droplet using a multiple-relaxation-time lattice Boltzmann method, Comput. Fluids 40 (2011) 156-171.

[49] H. Liu, A. J. Valocchi, Y. Zhang, Q. Kang, Phase-field-based lattice Boltzmann finite-difference model for simulating thermocapillary flows, Phys. Rev. E 87 (2013) 013010. 
[50] K. Connington, T. Lee, Lattice Boltzmann simulations of forced wetting transitions of drops on superhydrophobic surfaces, J. Comput. Phys. 250 (2013) 601-615.

[51] H. Ding, P. D. M. Spelt, Wetting condition in diffuse interface simulations of contact line motion, Phys. Rev. E 75 (2007) 046708.

[52] M. Geier, A. Fakhari, T. Lee, Conservative phase-field lattice Boltzmann model for interface tracking equation, Phys. Rev. E 91 (2015) 063309.

[53] P. Lallemand, L.-S. Luo, Theory of the lattice Boltzmann method: Dispersion, dissipation, isotropy, Galilean invariance, and stability, Phys. Rev. E 61 (2000) 6546.

[54] A. Kumar, Isotropic finite-differences, J. Comput. Phys. 201 (2004) $109-118$.

[55] O. Filippova, D. Hänel, Grid refinement for lattice-BGK models, J. Comput. Phys. 147 (1998) 219-228.

[56] R. Mei, L.-S. Luo, W. Shyy, An accurate curved boundary treatment in the lattice Boltzmann method, J. Comput. Phys. 155 (1999) 307-330.

[57] M. Bouzidi, M. Firdaouss, P. Lallemand, Momentum transfer of a Boltzmann-lattice fluid with boundaries, Phys. Fluids 13 (2001) 3452.

[58] D. Yu, R. Mei, W. Shyy, A unified boundary treatment in lattice Boltzmann method, AIAA (2003) 0953. 
[59] A. J. C. Ladd, Numerical simulations of particulate suspensions via a discretized Boltzmann equation. Part 1. Theoretical foundation, J. Fluid Mech. 271 (1994) 285.

[60] D. J. Tritton, Experiments on the flow past a circular cylinder at low reynolds numbers, J. Fluid Mech. 6 (1959) 547-567.

[61] E. Berger, R. Wille, Periodic flow phenomena, Annu. Rev. Fluid Mech. 4 (1972) 313.

[62] M. Braza, P. Chassaing, H. H. Minh, Numerical study and physical analysis of the pressure and velocity fields in the near wake of a circular cylinder, J. Fluid Mech. 165 (1986) 79-130.

[63] C. H. K. Williamson, Defining a universal and continuous StrouhalReynolds number relationship for the laminar vortex shedding of a circular cylinder, Phys. Fluid 31 (1988) 2742.

[64] C. H. K. Williamson, Vortex dynamics in the cylinder wake, Annu. Rev. Fluid Mech. 28 (1996) 477-539.

[65] X. He, G. D. Doolen, Lattice Boltzmann method on a curvilinear coordinate system: Vortex shedding behind a circular cylinder, Phys. Rev. E 56 (1997) 434.

[66] C. Liu, X. Zheng, C. H. Sung, Preconditioned multigrid methods for unsteady incompressible flows, J. Comput. Phys. 139 (1998) 35-57.

[67] D. Calhoun, A Cartesian grid method for solving the two-dimensional 
streamfunction-vorticity equations in irregular regions, J. Comput. Phys. 176 (2002) 231-275.

[68] H. Haddadi, S. Shojaei-Zadeh, J. F. Morris, Lattice-Boltzmann simulation of inertial particle-laden flow around an obstacle, Phys. Rev. Fluids 1 (2016) 024201.

[69] Q. Zu, X. He, On pressure and velocity boundary conditions for the lattice Boltzmann BGK model, Phys. Fluids 9 (1997) 1591.

[70] X. He, L.-S. Luo, lattice Boltzmann model for the incompressible NavierStokes equation, J. Stat. Phys. 88 (1997) 927.

[71] R. Mei, D. Yu, W. Shyy, L.-S. Luo, Force evaluation in the lattice Boltzmann method involving curved geometry, Phys. Rev. E 65 (2002) 041203.

[72] D. Richter, G. Iaccarino, E. S. G. Shaqfeh, Simulations of threedimensional viscoelastic flows past a circular cylinder at moderate Reynolds numbers, J. Fluid Mech. 651 (2010) 415-442.

[73] L. S. Hung, S. C. Yao, Experimental investigation of the impaction of water droplets on cylindrical objects, Int. J. Multiphase Flow 25 (1999) $1545-1559$.

[74] A. Hardalupas, A. M. K. P. Taylor, J. H. Wilkins, Experimental investigation of submillimetre droplet impingement on to spherical surfaces, Int. J. Heat Fluid Flow 20 (1999) 477-485. 
771 [75] S. Bakshi, I. V. Roisman, C. Tropea, Investigations on the impact of a 772 drop onto a small spherical target, Phys. Fluids 19 (2007) 032102.

773 [76] G. Liang, Y. Guo, Y. Yang, S. Guo, S. Shen, Special phenomena from 774 a single liquid drop impact on wetted cylindrical surfaces, Exp. Therm. $775 \quad$ Fluid Sci. 51 (2013) 18-27.

[77] D. Zhang, K. Papadikis, S. Gu, Application of a high density ratio lattice-Boltzmann model for the droplet impingement on flat and spherical surfaces, Int. J. Therm. Sci. 84 (2014) 75-85.

[78] A. L. Yarin, Drop impact dynamics: Splashing, spreading, receding, bouncing ..., Ann. Rev. Fluid Mech. 38 (2006) 159-192.

[79] P. Yue, C. Zhou, J. J. Feng, Sharp-interface limit of the Cahn-Hilliard model for moving contact lines, J. Fluid Mech. 645 (2010) 279-294. 